University of Nebraska - Lincoln

DigitalCommons@University of Nebraska - Lincoln

Faculty Publications from the Department of Engineering Mechanics

Mechanical \& Materials Engineering,

Department of

2009

\title{
Real-time depth sectioning: Isolating the effect of stress on structure development in pressure-driven flow
}

\author{
Lucia Fernandez-Ballester \\ University of Nebraska - Lincoln, lucia.fernandez@unl.edu \\ Derek W. Thurman \\ California Institute of Technology \\ Julia A Kornfield \\ California Institute of Technology, jak@cheme.caltech.edu
}

Follow this and additional works at: https://digitalcommons.unl.edu/engineeringmechanicsfacpub

Part of the Mechanical Engineering Commons

Fernandez-Ballester, Lucia; Thurman, Derek W.; and Kornfield, Julia A, "Real-time depth sectioning: Isolating the effect of stress on structure development in pressure-driven flow" (2009). Faculty Publications from the Department of Engineering Mechanics. 67.

https://digitalcommons.unl.edu/engineeringmechanicsfacpub/67

This Article is brought to you for free and open access by the Mechanical \& Materials Engineering, Department of at DigitalCommons@University of Nebraska - Lincoln. It has been accepted for inclusion in Faculty Publications from the Department of Engineering Mechanics by an authorized administrator of DigitalCommons@University of Nebraska - Lincoln. 


\title{
Real-time depth sectioning: Isolating the effect of stress on structure development in pressure-driven flow
}

\author{
Lucia Fernandez-Ballester, ${ }^{\text {a) }}$ Derek W. Thurman, ${ }^{\text {b) }}$ and Julia A. Kornfield \\ Division of Chemistry and Chemical Engineering, California Institute of \\ Technology, Pasadena, California 91125
}

(Received 24 February 2009; final revision received 10 June 2009)

\begin{abstract}
Synopsis
Transient structure development at a specific distance from the channel wall in a pressure-driven flow is obtained from a set of real-time measurements that integrate contributions throughout the thickness of a rectangular channel. This "depth sectioning method" retains the advantages of pressure-driven flow while revealing flow-induced structures as a function of stress. The method is illustrated by applying it to isothermal shear-induced crystallization of an isotactic polypropylene using both synchrotron $\mathrm{x}$-ray scattering and optical retardance. Real-time, depth-resolved information about the development of oriented precursors reveals features that cannot be extracted from ex-situ observation of the final morphology and that are obscured in the depth-averaged in-situ measurements. For example, at $137^{\circ} \mathrm{C}$ and at the highest shear stress examined (65 kPa), oriented thread-like nuclei formed rapidly, saturated within the first $7 \mathrm{~s}$ of flow, developed significant crystalline overgrowth during flow and did not relax after cessation of shear. At lower stresses, threads formed later and increased at a slower rate. The depth sectioning method can be applied to the flow-induced structure development in diverse complex fluids, including block copolymers, colloidal systems, and liquid-crystalline polymers. (C) 2009 The Society of Rheology. [DOI: 10.1122/1.3164970]
\end{abstract}

\section{INTRODUCTION}

Pressure-driven flows through a channel are widely used for rheological measurements (e.g., capillary rheometers) and materials processing (e.g., extrusion). These flows are also used for in-situ studies of flow-induced structure using rheo-optical and rheo-X-ray measurements [Liedauer et al. (1993); Seki et al. (2002); Bent et al. (2003); Kumaraswamy et al. (2004); Carreras et al. (2006)]. Pressure-driven flow is particularly attractive for studying transient structure development as a function of stress because it affords a facile and robust way to impose a specific wall shear stress, and sudden inception and cessation of shear are easily implemented. In particular, when an experiment demands a rapid imposition and release of a very high shear stress, a pressure-driven flow eliminates some of the challenges associated with sliding plate and torsional geometries (tool compliance, tool inertia, and sample edge fracture, among other difficulties). A drawback of

\footnotetext{
${ }^{a)}$ Present address: Netherlands Organization for Scientific Research (NWO), DUBBLE at ESRF, BP 220, F-38043 Grenoble CEDEX, France.

${ }^{b}$ Present address: ExxonMobil Chemical Co., Baytown Technology and Engineering Complex, 5200 Bayway Drive, Baytown, Texas 77520.
} 
the pressure-driven flow is that a range of shear stresses is probed during each experiment. Therefore, we offer an approach that combines the best features of rheometric flows, which unambiguously reveal structure development at a given stress, and those of pressure-driven flows.

The "depth sectioning method" presented here isolates the real-time structure at a given depth in the channel (i.e., at a certain level of shear stress). The method takes advantage of the linear variation in the local shear stress from the wall to the center of a rectangular slit channel to extract the stress dependence of the effect of flow on structure formation. Measurements made using two different wall shear stresses, $\sigma_{w, 1}$ and $\sigma_{w, 2}$, are used to compute the incremental contribution to the observable of interest due to stresses from $\sigma_{w, 1}$ to $\sigma_{w, 2}$ by appropriately subtracting results for the lower stress experiment from the higher stress one. We demonstrate the approach by applying it to crystallization of polymers during and after an interval of shear. It may be used more broadly, for example, in studies of shear-induced alignment of block copolymers or shear-induced morphology in polymer blends.

Flow-induced crystallization provides an instructive example for "depth sectioning" for three reasons: (1) the stress dependence is highly nonlinear, (2) it involves transient measurements during inception and cessation of flow, and (3) it illustrates how the control of conditions during flow permits a depth-resolved analysis of structure development after cessation of flow as well. Flow-induced crystallization can result in an oriented morphology often referred to as a "shish-kebab" due to its central core decorated by crystalline overgrowth [Pennings and Kiel (1965)]. Thread-like precursors ("shish") are at the heart of flow-induced crystallization, as they are responsible for the dramatic changes in kinetics of crystallization and in the final morphology obtained. The creation of these precursors depends strongly on stress. Consequently, pronounced stratified morphologies are frequently observed when pressure-driven flows are imposed on the melt, including isothermal channel flows that are considered here.

A qualitative model put forward by Janeschitz-Kriegl and co-workers [Liedauer et al. (1995)] captures the sequence of events that occur during a brief interval of isothermal pressure-driven flow starting with a fully amorphous, fully relaxed, and subcooled polymer melt [Fig. 1(a)]. First, point-like nuclei form $\left(t=t_{1}\right)$ at a rate that depends on the local shear stress (i.e., the number of nuclei per unit volume increases with distance from the channel center). With prolonged shear, thread-like precursors may grow from these pointlike nuclei if the imposed stress is sufficiently high: shish form prolifically above a critical shear stress $\left[t>t_{2}\right.$ in Fig. 1(a)], which depends on the material [Seki et al. (2002)]. These threads continue to grow in length during flow (e.g., $t=t_{3}$ ) until, if shear is sustained long enough, saturation is reached [illustrated by the region adjacent to the wall, unchanged between $t=t_{3}$ and $t_{4}$ in Fig. 1(a)]. Time-resolved information on the appearance of a detectable population of shish, their progressive increase in length, and their saturation in thin "slices" $(<50 \mu \mathrm{m}$ thick) at different distances from the channel wall during flow is provided by depth sectioning of the optical retardance measured using a beam propagating through the thickness of the channel (Fig. 2).

Precursors present at the moment that shear is stopped dictate the rate and morphology of crystallization that ensues quiescently. For example, suppose that shear ceased at time $t$ in Fig. 1(a), leading to the morphology development illustrated in Fig. 1(b). Each of the thread-like precursors nucleates highly oriented lamellae ("kebabs") that grow radially outward, advancing at the quiescent linear growth rate until they impinge with lamellae growing from neighboring precursors. The high nucleation surface of the thread-like precursors accelerates crystallization by orders of magnitude relative to the quiescent case [Liedauer et al. (1993); Kumaraswamy et al. (2000)]. Where thread-like precursors 
a)

$\sigma=0$ (center plane)

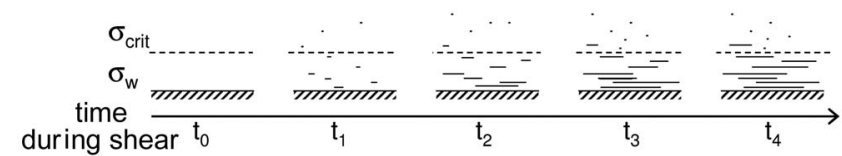

b)

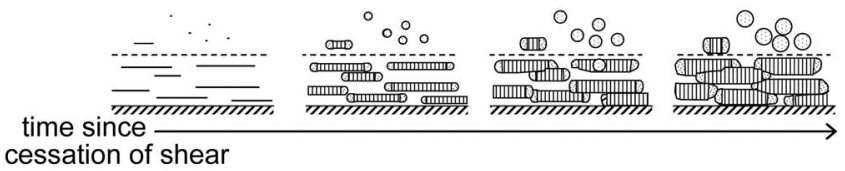

FIG. 1. Schematic of (a) the sequence of events during shear and (b) their consequences for morphology development after the cessation of shear under isothermal conditions. During shear (a), flow-induced formation of point-like precursors begins; if shear is sustained, thread-like precursors grow from the point-nuclei where $\sigma>\sigma_{\text {crit }}$, a critical shear stress; thread-like precursors may reach saturation at long enough shearing time. A few isolated thread-like precursors may form below the critical shear stress. Precursors that exist at the moment that shear is stopped govern the morphology that grows quiescently after cessation of flow (b): a spherullitic morphology grows in zones of point-like precursors, a "sausage" morphology develops where point-like precursors coexist with few thread-like ones, and a highly oriented morphology develops where thread-like precursors are predominant.

are close to each other (less than a few microns apart), the oriented lamellae impinge before they can diverge, producing a highly oriented "skin." Where thread-like precursors are sparse, kebabs are able to grow over long distances prior to impingement, producing "sausages"- birefringent elongated super-structures that are microns wide (Fig. 3 micrographs). Strata with sausages have a lower degree of orientation than the skin [FernandezBallester (2007)]. Regions that only contain point-like precursors develop a spherullitic morphology with a variation in spherullite size with the distance from the wall that is dictated by the stress dependence of point-like precursor formation. Depth sectioning of real-time synchrotron x-ray patterns measured using a beam propagating through the thickness of the channel (Fig. 2) is used to obtain depth-resolved information on the orientation distribution of crystallites, the extent of crystallization, and the time at which impingement occurs.

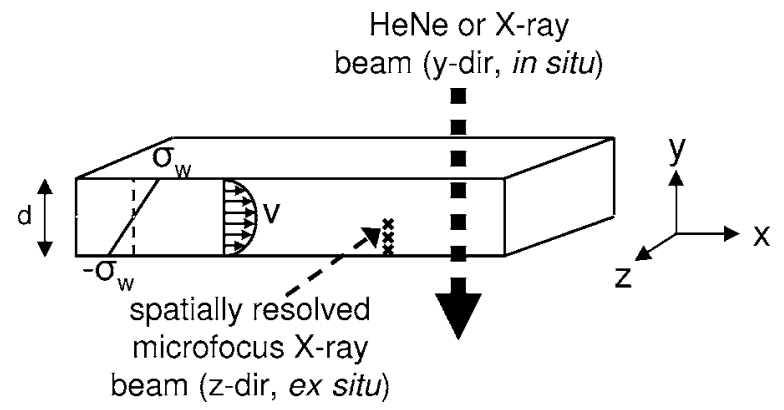

FIG. 2. Diagram of rectangular slit channel for pressure-driven flow showing the flow-velocity gradient plane ( $x y)$ and the linear stress profile varying from $-\sigma_{w}$ at $y=-d / 2$ to $\sigma_{w}$ at $y=d / 2$. The orientation of the probe beam is along the velocity gradient direction $(y)$ for the real-time optical and $\mathrm{x}$-ray measurements and is along the vorticity direction $(z)$ for the $e x$-situ microfocus WAXD measurements. 
Our flow-induced crystallization apparatus [Kumaraswamy et al. (1999b)] uses the short-term shearing protocol developed by Janeschitz-Kriegl [Eder et al. (1990); Janeschitz-Kriegl and Eder (1990); Liedauer et al. (1995); Eder and Janeschitz-Kriegl (1997)]: a brief and intense shear pulse is imposed on the polymer melt using pressuredriven flow, and the real-time development of crystallization is monitored with a variety of in-situ probes. Real-time measurements using optical and x-ray beams that traverse the thickness of the channel (Fig. 2) record the structure development in an integrated sense, throughout the depth of the channel.

To apply the depth sectioning method, we perform a series of experiments $(i$ $=1,2, \ldots, n)$ applying successively lower wall shear stress $\left(\sigma_{w, 1}>\sigma_{w, 2}>\ldots \sigma_{w, n}\right)$ while keeping the other parameters of the experiment fixed (the shear pulse duration and the isothermal temperature). For the $i$ th experiment in the series, the resulting observable at any moment in time reflects an average over structures induced by shear stresses from zero at the center of the channel to $\sigma_{w, i}$ at the wall. This same range of structures is present at time $t$ in the interior of the samples subjected to greater wall shear stress. Simply rescaling the measurement made in the experiment using the lower wall shear stress $\sigma_{w, i}$ and subtracting it from the measurement performed with $\sigma_{w, i-1}$ provides the contribution due to the interval of stress between $\sigma_{w, i}$ and $\sigma_{w, i-1}$. We show that this depth sectioning method is rigorously founded for a number of different observables used to study complex fluids, including birefringence and dichroism (when depolarization is negligible) and light, neutron and x-ray scattering patterns (when in the single-scattering regime). Depth sectioning of the present optical and x-ray measurements leads to new findings regarding the formation of oriented precursors during flow-induced crystallization.

\section{DEPTH SECTIONING METHOD}

The depth sectioning method relies on the fact that the stress profile varies linearly from $\sigma_{w}$ to zero in a pressure-driven laminar flow through a slit channel (Fig. 2). The linear profile arises directly from a force balance-independent of the viscoelastic properties of the material in the channel and the velocity boundary condition at the walls. Consequently, if the wall shear stress is known, the shear stress $\sigma_{x y}$ at any distance $y$ from the center plane is known. Provided that the structural consequences of the imposed stress arise from local physical processes, the transient structure development in a fluid element is specified by its stress history (all other conditions held fixed, such as thermal history, material composition, etc.). Many fluids have a characteristic length scale beyond which structure and dynamics of the fluid is independent of neighboring fluid elements (e.g., two phase flows, block copolymers, and microemulsions). The present method is restricted in its spatial resolution to length scales large enough that the fluid of interest satisfies the assumption of local response. Also, the method is restricted to structural observables that accumulate additively as the beam propagates through each differential thickness of the sample. Fortunately, under a wide range of conditions, many rheooptical, rheo-small-angle neutron scattering, and rheo-X-ray methods satisfy the additivity requirement. Together, the robust relationship between $y$ and $\sigma_{x y}(y)$, local response and additive observables allow us to isolate the contribution from a given "slice" of material (i.e., from certain range of shear stress) using a set of experiments for which different wall shear stresses have been applied.

To illustrate, we present sets of four flow-induced crystallization experiments in which successively lower wall shear stresses $\sigma_{w}$ were imposed for a fixed shearing time $t_{s}$ and identical crystallization temperature $T_{x}$ [Fig. 3(a)]. The plane of observation in these 


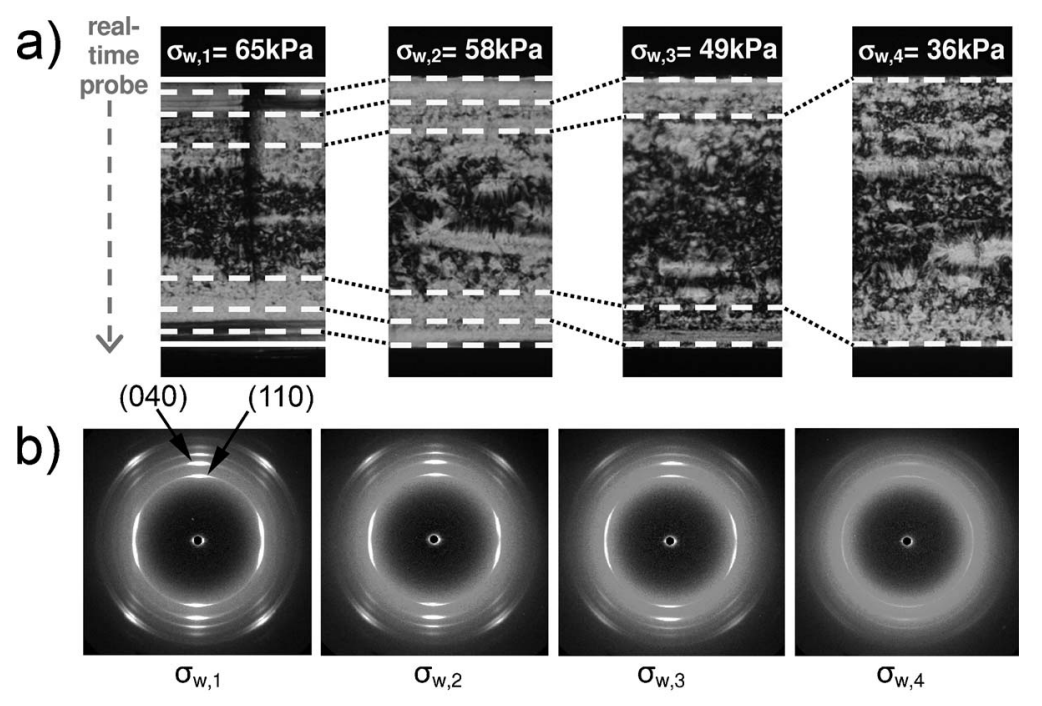

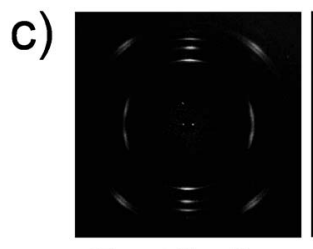

$\sigma_{\mathrm{w}, 1}<\sigma<\sigma_{\mathrm{w}, 2}$

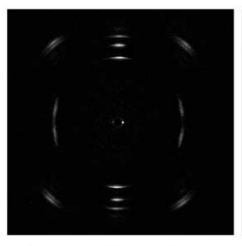

$\sigma_{\mathrm{w}, 2}<\sigma<\sigma_{\mathrm{w}, 3}$

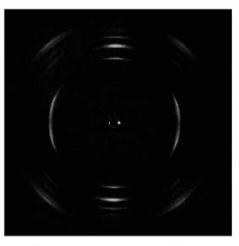

$\sigma_{w, 3}<\sigma<\sigma_{w, 4}$

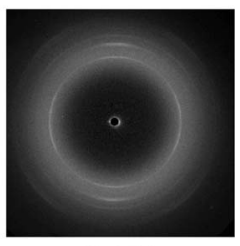

$\sigma<\sigma_{\mathrm{w}, 4}$

FIG. 3. (a) Ex-situ polarized optical micrographs of samples sheared at $137^{\circ} \mathrm{C}$ for $12 \mathrm{~s}$, with successively smaller $\sigma_{w}$. Dashed lines show corresponding shear stresses. (b) Corresponding real-time WAXD patterns acquired $1145 \mathrm{~s}$ after the cessation of flow for each experiment. (c) Resulting WAXD patterns after applying the depth sectioning method to the real-time WAXD shown in (b). Flow is along the horizontal direction. The (110), (040), (130), (111), and (-131/041) reflections of the $\alpha$ morph of iPP are observed in these patterns.

ex-situ micrographs corresponds to the $x y$ plane (Fig. 2). The strong effects of stress on structure development during and after flow are manifested in the final solid state morphology. Moving from the highest imposed stress $\left(\sigma_{w, 1}=65 \mathrm{kPa}\right)$ to the lowest $\left(\sigma_{w, 4}\right.$ $=36 \mathrm{kPa}$ ), the local morphology in successive samples corresponds when compared at matched stress. For example, the morphology near the wall in the $\sigma_{w, 4}$ specimen is found at increasing distance from the wall as $\sigma_{w}$ increases, at a location where the local stress equals $\sigma_{\mathrm{w}, 4}$. In other words, consider the position of the boundary with the fine-grained interior: it shifts from the wall (i.e., $y=d / 2)$ to $y=(d / 2)\left(\sigma_{w, 4} / \sigma_{w, 3}\right)$, then to $y=(d / 2)$ $\times\left(\sigma_{w, 4} / \sigma_{w, 2}\right)$, and to $y=(d / 2)\left(\sigma_{w, 4} / \sigma_{w, 1}\right)$ [Fig. 3(a), right to left]. On physical grounds, this simply reflects the fact that structure development is local (discussed further below).

We analyze real-time observables accordingly. In-situ measurements are obtained using a beam directed along the $y$ axis (Fig. 2), providing an average throughout the depth of each sample. Consider an appropriate real-time observable $I\left(\sigma_{w}\right)$ that has been measured for two experiments, with wall shear stresses of $\sigma_{w, 1}$ and $\sigma_{w, 2}$. The contribution to $I$ due to the outermost slice of material in the experiment with greater wall shear stress (i.e., to the stress range between $\sigma_{w, 2}$ and $\sigma_{w, 1}$ ) is equal to

$$
\Delta I\left(\sigma_{w, 1}, \sigma_{w, 2}\right)=I\left(\sigma_{w, 1}\right)-I\left(\sigma_{w, 2}\right)\left(\sigma_{w, 2} / \sigma_{w, 1}\right) .
$$

The rescaling of $I\left(\sigma_{w, 2}\right)$ by the stress ratio is analogous to both the familiar Rabinowitsch correction to evaluate the wall shear rate in a capillary rheometer and the procedure to 
calculate the birefringence at the wall of a slit rheometer [Wales (1969)]. This scheme can be repeated by performing experiments at successively lower wall shear stresses, thus isolating the signal arising from slices at different depths corresponding to ranges of shear stress $\sigma_{i+1}<\sigma<\sigma_{i}$. This reasoning holds at each moment in time, during and after shear, provided that the sample, the shearing time, and the temperature are the same for all experiments.

For example, the real-time wide-angle x-ray diffraction (WAXD) patterns observed 30 min after cessation of shear for each of the $\sigma_{w . i}$ above [Fig. 3(b)] show the superimposed contributions of the spherullitic core, the partially oriented "sausage" layer, and the highly oriented skin. The contribution of each differential layer of sample to the overall diffraction pattern is additive. Therefore, pixel-by-pixel we subtract the "interior" contribution $\left(\sigma_{w, i+1} / \sigma_{w, i}\right) I_{w, i+1}$ from the scattering patterns $I_{w, i}$ for a given experimental time [Fig. 3(c)]. To compare the results obtained from depth slices of different thicknesses, each one is normalized by the total thickness of the depth sections, so that an average quantity is extracted.

For the present example, we first demonstrate the local character of flow-induced crystallization. Then we consider the interaction of the light and x-rays with the sample to establish the additivity of each observable.

\section{A. Flow-induced crystallization as a local phenomenon}

Depth sectioning is limited to length scales greater than the structural length scale of the material. In the present case of flow-induced crystallization, we restrict depth sections to $23 \mu \mathrm{m}$ or more. Previous studies have shown that the distance between nuclei sets the length scale beyond which flow-induced crystallization is a local phenomenon. For example, the highly oriented skin [Fig. 3(a)] is templated by thread-like precursors that are less than a micron apart [Kumaraswamy et al. (2000); Kornfield et al. (2002)]. Therefore, volume elements in the oriented skin separated by more than a few microns crystallize independently of each other; this screening also prevents nucleation on the wall from influencing volume elements that are more than a few microns from the wall [Thurman (2005)]. While the textures in the highly oriented skin and in the fine-grained layer are consistently on length scales $\leq 5 \mu \mathrm{m}$, the sausage layer may sometimes violate the separation of length scales (crystallites growing outward from the core of a sausage in one depth section might propagate far enough to contribute to crystallization in an adjacent depth section). The real-time measurements here, nevertheless, satisfy local response because the lamellae have not had sufficient time to grow more than $5 \mu \mathrm{m}$ prior to the conclusion of the in-situ measurements (1200 s). Since the "thickness" of the depth sections $(\geq 23 \mu \mathrm{m})$ is greater than this, we can treat the crystallization in any particular depth section as a function of local conditions (e.g., the stress and thermal history).

\section{B. Additive contributions to optical and x-ray observables}

To understand the regime of validity of the depth sectioning analysis, consider the conditions under which the key observables used to characterize the flow-induced structure can be analyzed as the sum of contributions from each depth in the sample.

\section{Polarimetric observables}

As polarized light passes through a complex fluid (e.g., a polymer melt in which crystallization is beginning), it is altered by the anisotropic retardance (due to intrinsic and form birefringence) and anisotropic attenuation (dichroism) due to absorbance or scattering (e.g., by structures that have anisotropic shape and optical properties). The 
polarization state represented by the Jones vector A [Fuller (1995)] changes from its incident state $\mathbf{A}_{o}$ to its exiting state $\mathbf{A}_{d}$ after transmission through a sample of path length $d$. Considering only linear optical phenomena (compatible with the Jones matrix formalism), neglecting depolarization (which can occur due to multiple scattering if the material becomes highly turbid) and approximating the system as a succession of optical elements, the cumulative change in the polarization of the beam traversing $m$ optical elements of thickness $\Delta y=d / m$, each described by its particular Jones matrix $\mathbf{J}_{i}$, is given by [Fuller (1995)]

$$
\mathbf{A}_{d}=\mathbf{J}_{m} \cdot \mathbf{J}_{m-1} \cdot \ldots \cdot \mathbf{J}_{2} \cdot \mathbf{J}_{1} \cdot \mathbf{A}_{0} .
$$

The Jones matrices take a particularly simple form for shear flow and for light propagating down the velocity gradient direction (Fig. 2). The complex refractive index tensor $\mathbf{n}=\mathbf{n}^{\prime}+i \mathbf{n}^{\prime \prime}\left[\right.$ Fuller (1995)] is simplified by symmetry about the $x y$ plane (i.e., $n_{x z}=n_{y z}$ $=0)$,

$$
\mathbf{n}=\left[\begin{array}{ccc}
n_{x x} & n_{x y} & 0 \\
n_{x y} & n_{y y} & 0 \\
0 & 0 & n_{z z}
\end{array}\right]
$$

Furthermore, the light propagating along the $y$ axis is affected by the optical anisotropy in the $x z$ plane, in which the principal axes for both dichroism $\left(\Delta n^{\prime \prime}=n_{x x}^{\prime \prime}-n_{z z}^{\prime \prime}\right)$ and birefringence $\left(\Delta n^{\prime}=n_{x x}^{\prime}-n_{z z}^{\prime}\right)$ coincide with the $x$ and $z$ axes. Consequently, the Jones matrix $\mathbf{J}_{\Delta y}(y)$ for each differential element of thickness $\Delta y$ is diagonal,

$$
\mathbf{J}_{\Delta y}=\left[\begin{array}{cc}
e^{i\left(2 \pi n_{x x} \Delta y / \lambda\right)} & 0 \\
0 & e^{i\left(2 \pi n_{z z} \Delta y / \lambda\right)}
\end{array}\right]=e^{i\left(2 \pi n_{x x} \Delta y / \lambda\right)}\left[\begin{array}{cc}
1 & 0 \\
0 & e^{-i \delta}
\end{array}\right],
$$

where $\delta(y)=\delta^{\prime}(y)+i \delta^{\prime}(y)$, with retardance

$$
\delta^{\prime}=\frac{2 \pi \Delta n^{\prime} \Delta y}{\lambda}
$$

and extinction

$$
\delta^{\prime \prime}=\frac{2 \pi \Delta n^{\prime \prime} \Delta y}{\lambda}
$$

Since all slices have the same principal axes, the expression for the change in polarization upon transmission through $m$ slices is simply,

$$
\mathbf{A}_{m}=\prod_{i=1}^{m} e^{\left(2 \pi n_{x x}\left(y_{i}\right) \Delta y / \lambda\right)}\left[\begin{array}{cc}
1 & 0 \\
0 & e^{-i \delta\left(y_{i}\right)}
\end{array}\right] \mathbf{A}_{o} .
$$

Written in terms of the mean refractive index, $\langle n\rangle=\sum n_{x x}(y) \Delta y / d$, and considering the limit $\Delta y \rightarrow 0$,

$$
\mathbf{A}_{d}=e^{(2 \pi\langle n\rangle d / \lambda)}\left[\begin{array}{cc}
1 & 0 \\
0 & \exp \left[-i \int_{-d / 2}^{+d / 2} \delta(y) d y\right]
\end{array}\right] \mathbf{A}_{o}
$$

describes the cumulative change in polarization of light upon propagating through the sample, provided the sample does not cause significant depolarization of light. Thus, the 
retardance (and dichroism) accumulates additively as the beam propagates through the sample in the $y$-direction (Fig. 2).

In the example of flow-induced crystallization, the evolution of structure after cessation of flow is as important as the transient structure development during flow. Therefore, it is significant that the symmetry of shear flow governs the orientation of the principal axes not only during shear flow but also for the optical anisotropy that arises from ongoing structure development (e.g., oriented crystallization) after shear has stopped. Symmetry about the $x y$ plane persists during structure evolution in complex fluids after cessation of shear (e.g., growth of crystallites, relaxation/breakup/coalescence of droplets, or coarsening of domains in an ordered block copolymer or a liquid crystal) provided the length scale of interest is large relative to the texture in the material (e.g., cyllindrulite/ spherullite, droplet, or domain size). Thus, the principal axes of the anisotropy in the $x z$ plane continue to coincide with the $x$ and $z$ directions.

Polarimetry measurements in the present example are only made during early stages, before turbidity has become significant. Therefore, there is little absorption or scattering by the sample, so neither dichroism nor depolarization is significant. Consequently, the depth sectioning method here only involves the retardance contribution of each depth slice to the final signal [i.e., Eq. (1) is applied with $I$ replaced by $\delta^{\prime}$ ].

\section{Scattering measurements}

In the case of WAXD and small-angle X-ray scattering (SAXS) under our experimental conditions, the scattered intensity is the sum of that due to each depth slice whenever the kinematic theory of diffraction and the Fraunhofer approximation apply [Roe (2000)]. ${ }^{1}$ As is typically the case, the thickness of our sample is thin enough and its scattering power is low enough that most photons pass through our sample unscattered, satisfying the assumption that the scattered $\mathrm{x}$-rays interfere with other scattered $\mathrm{x}$-rays but do not undergo any further interactions with other atoms. In addition, the present sample is very thin compared to the observation distance (as usual): here the total sample thickness $(0.5$ $\mathrm{mm})$ is much less than the camera length $(>170 \mathrm{~mm}$ for WAXD and $>6000 \mathrm{~mm}$ for SAXS measurements). Whenever these theoretical approximations hold for the whole sample, they also hold for each "depth section" in it.

As long as a rheo-X-ray experiment conforms to single-scattering and Fraunhofer diffraction, the angular distribution of the x-ray scattering amplitude is linearly related (by Fourier transformation) to the electron density of the scattering substances [Roe (2000)]. For scatterers that are numerous and more or less continuously distributed in the sample,

$$
A(\mathbf{q})=\int_{V} \rho(\mathbf{r}) e^{-i \mathbf{q} \cdot \mathbf{r}} d \mathbf{r}
$$

where $A(\mathbf{q})$ is the amplitude of the scattered wave normalized by the incident $A_{o}, \mathbf{q}$ is the scattering vector, $\rho(\mathbf{r})$ is the scattering length density distribution, $d \mathbf{r}$ is a volume element, and the integration is performed over the illuminated sample volume $V$. The scattered intensity $I$ is related to $A(\mathbf{q})$ by

\footnotetext{
${ }^{1}$ Exceptions to this approximation can occur, for example, in a large, perfect crystal, in which a diffracted ray could be diffracted many times in different regions before finally leaving the crystal (dynamic theory of x-ray diffraction).
} 


$$
I(\mathbf{q})=\left\langle|A(\mathbf{q})|^{2}\right\rangle=\left\langle\left|\int_{V} \rho(\mathbf{r}) e^{-i q \mathbf{r}} d \mathbf{r}\right|^{2}\right\rangle=\int_{V} \Gamma_{\rho}(\mathbf{r}) e^{-i q \mathbf{r}} d \mathbf{r},
$$

where $\Gamma_{\rho}$ is the autocorrelation function of $\rho(\mathbf{r})$. Since the thickness of interest for depth sectioning $(>10 \mu \mathrm{m})$ is much greater than the length scale of structural periodicities that give rise to WAXD and SAXS, the derivation above applies for each slice $m$ (with volume $V_{m}$ ) independently, and the scattering is simply the sum of their contributions. This reasoning applies equally to light scattering (in the single-scattering regime) and to neutron and x-ray scattering. In the present flow-induced crystallization example, Eq. (1) is applied with $I$ being the two-dimensional array of measured WAXD intensities at each measuring time.

\section{EXPERIMENTAL}

\section{A. Materials}

A bimodal blend containing 1 wt $\%$ of very high molecular weight isotactic polypropylene $\left(M_{w}=3500 \mathrm{~kg} / \mathrm{mol}, \mathrm{PDI}=1.8,[\mathrm{mmmm}]>98 \%\right)$ in a lower molecular weight isotactic polypropylene (iPP) $\operatorname{resin}\left(M_{w}=186 \mathrm{~kg} / \mathrm{mol}, \mathrm{PDI}=2.3,[\mathrm{mmmm}]>96 \%\right)$ was prepared by fully dissolving the desired amount of each species in hot xylene under inert atmosphere then quickly precipitating into cold methanol. The resulting "fluff" was filtered, washed with methanol, and then sprayed with an acetone solution of antioxidants to introduce $2000 \mathrm{ppm}$ of Irganox 1010 and Irgafos 168. Then the polymer was dried at $80{ }^{\circ} \mathrm{C}$ under vacuum for several days. Both polymers were provided by Dow Chemical and were synthesized using single-site catalysts. The binary blend was designed to investigate the effect of small, but known, concentrations of long chains on flow-induced crystallization [Fernandez-Ballester (2007)].

\section{B. Apparatus and protocol}

The flow-induced crystallization was performed in a short-term shearing apparatus described previously [Kumaraswamy et al. (1999b)]. The experimental protocol was as follows. First the flow cell was filled with the polymer melt at a low wall shear stress at $215^{\circ} \mathrm{C}$. It was held at $215^{\circ} \mathrm{C}\left(T_{\text {erase }}\right)$ for $5 \mathrm{~min}$ to erase the previous flow history and fully relax the melt [Seki et al. (2002)]. Then, it was cooled to $137{ }^{\circ} \mathrm{C}\left(T_{x}\right)$ and a shear pulse with a specific wall shear stress $\left(\sigma_{w}\right)$ was applied for a shearing time $\left(t_{s}\right)$ of either 7 or $12 \mathrm{~s}$. Subsequently, the polymer was held isothermally at $137^{\circ} \mathrm{C}$, and the progress of crystallization was monitored with rheo-optical and rheo-WAXD techniques. At the end of the experiment, the flow cell was cooled to room temperature (over $\sim 10 \mathrm{~min}$ ), and the sample was removed for ex-situ characterization. Groups of four experiments were performed with a series of $\sigma_{w}$ between 65 and $36 \mathrm{kPa}$.

\section{In-situ optical measurements}

Using a simple optical setup described elsewhere [Kumaraswamy et al. (1999b)], the intensities of polarized light transmitted through the sample and both crossed $\left(I_{\perp}\right)$ and parallel $\left(I_{\|}\right)$polarizers are simultaneously recorded. From the measured $I_{\perp} /\left(I_{\perp}+I_{\|}\right)$, the retardance $\delta^{\prime}$ of the sample at early times (when $\delta^{\prime}<\pi$ ) is computed, 


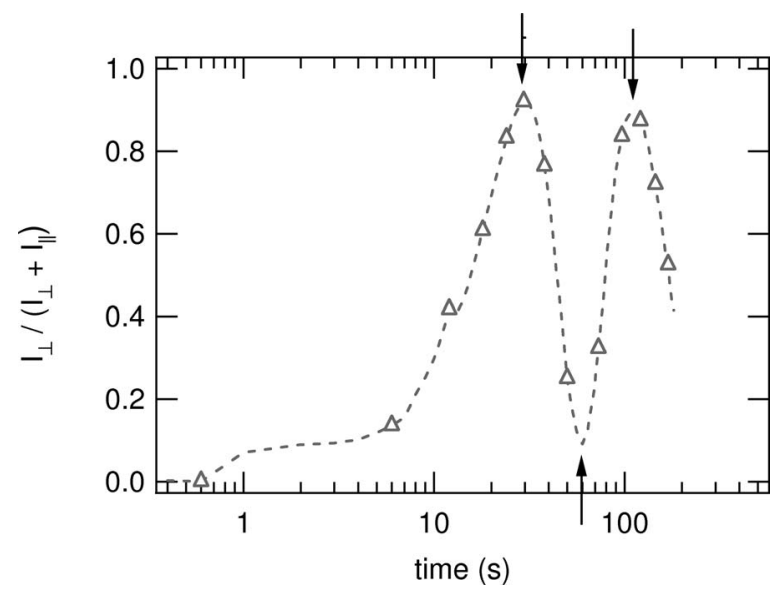

FIG. 4. Example of normalized intensity between crossed polars for the experiment with $\sigma_{w}=0.065 \mathrm{MPa}$ and $t_{s}=12 \mathrm{~s}$. The three arrows show the points in time where the retardance was computed by observing the successive maxima and minima in the intensity (of known retardance $\delta^{\prime}=\pi, 2 \pi, 3 \pi$ ). There is an observable depolarization (the maxima and minima do not reach 1 and 0 for complete transmission or extinction), but it is sufficiently small that extrema continue to very nearly coincide with integer orders in retardance (Hongladarom et al., 1993).

$$
\delta^{\prime}=2 \arcsin \sqrt{\frac{I_{\perp}}{I_{\perp}+I_{\|}}} .
$$

At later times (when $\delta^{\prime}>\pi$ ), we simply compute the points in time at which we observe the successive maxima and minima in the intensity and for which the corresponding retardance is known $\left(\delta^{\prime}=\pi, 2 \pi, 3 \pi\right.$, etc.). Burghardt and co-workers [Hongladarom et al. (1993)] determined in studies of spectrographic birefringence for liquid crystals that calculating birefringence from successive maxima and minima is adequate even when there is significant depolarization. In our case, there is some depolarization attained at later stages of the experiment but it is small (Fig. 4). Therefore, we use successive maxima and minima to estimate the time at which the retardance reaches the corresponding order $n \pi$.

Four experiments were performed for each of the two shearing times ( 7 and $12 \mathrm{~s}$ ), with the greatest value of the wall shear stress being $\sigma_{w, 1}=65 \mathrm{kPa}$. From the point of view of that highest stress experiment, the successively lower stresses $\sigma_{w, 2}=58, \sigma_{w, 3}=49$ and $\sigma_{w, 4}=43 \mathrm{kPa}$ correspond to distances of 27,62 and $85 \mu \mathrm{m}$ from the wall, respectively.

To isolate the contribution to the total retardance $\delta^{\prime}$ arising from each stress interval or, equivalently, each depth section, the retardance computed at each time $t$ for the experiment with $\sigma_{w, n+1}$ was rescaled by $\sigma_{w, n+1} / \sigma_{w, n}$ to account for the corresponding optical path [Fig. 3(a)] and subtracted from the retardance at $t$ for the experiment with $\sigma_{w, n}$ according to Eq. (1). For time points at which the retardance for the experiment at the greater stress $\sigma_{w, n}$ had already gone over orders, a linear interpolation between the values of $\delta^{\prime}$ corresponding to "peaks" and "valleys" was used to obtain values at each desired $t$. The average birefringence $\Delta n$ in each depth section $m$ was computed from its retardance using the known thickness $d_{m}$ of the particular slice and the wavelength $\lambda$ of light used for the measurement,

$$
\Delta n=\frac{\delta^{\prime} \lambda}{2 \pi d_{m}} .
$$




\section{In-situ x-ray measurements}

Real-time WAXD measurements were performed at the X27C beamline of the National Synchrotron Light Source, Upton, NY. The experimental setup is the same as used for flow birefringence measurements, except that kapton-sealed beryllium windows are used. The x-ray wavelength was $1.371 \AA$. A MARCCD camera was used as the detector

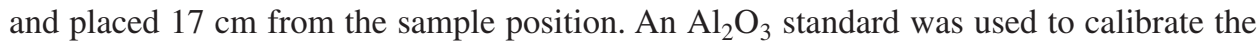
camera length. All acquired patterns were normalized by the incident intensity of the $\mathrm{x}$-ray source and by the acquisition time ( $5 \mathrm{~s}$ at early time and $30 \mathrm{~s}$ at later times). The background pattern corresponding to the empty cell was subtracted.

As above, groups of four experiments were performed for each shearing time ( 7 and $12 \mathrm{~s})$ with all parameters held fixed except for the wall shear stress. Again, the greatest wall shear stress used was $\sigma_{w, 1}=65 \mathrm{kPa}$. The successively lower wall shear stresses are not exactly the same as those used above in the optical experiments; nevertheless, we denote them by $\sigma_{w, 2}=58, \sigma_{w, 3}=49$, and $\sigma_{w, 4}=36 \mathrm{kPa}$, as shown in Fig. 3. The four WAXD patterns in Fig. 3(b) show the normalized background-subtracted data sets obtained in situ at $t=1145 \mathrm{~s}$ for $t_{s}=12 \mathrm{~s}$; those in Fig. 3(c) [analyzed according to Eq. (1)] isolate the scattered intensity due to specific depth sections and normalize it by the thickness of each section [Fig. 3(a)].

Using the four depth-sectioned data sets at each $t$, we analyzed the crystallographic reflections that correspond to the (110) and (040) planes of the $\alpha$ crystal morph of isotactic polypropylene to characterize (1) the growth of oriented crystallites using the azimuthal integral of the (110) reflections over a wedge about the equatorial direction, due to the oriented parent crystallites $A_{P, 110}$; (2) the degree of orientation using the azimuthal full width at half maximum (FWHM) of the equatorial peak, $\mathrm{FWHM}_{P, 110}$; and (3) the relative populations of parent crystallites and their epitaxial daughters. The amorphous contribution was subtracted prior to performing the azimuthal scans of intensity. Next, the unoriented crystalline contribution-estimated using the (040) reflection-was subtracted from the (110) intensity: because oriented parents and their epitaxial daughters share the same $b$ axis direction, both scatter intensity only in the equatorial region for the (040) plane, so the unoriented population can be more accurately determined. Azimuthal scans were fitted by Lorentzian peaks, and the area and FWHM for each one of them were computed. These were used to estimate the amounts of parent and daughter lamellae, applying the geometrical correction appropriate for their respective diffraction angles [Dean et al. (1998)].

\section{E. Ex-situ characterization}

Ex-situ sections of the flow-velocity gradient $(x y)$ plane were obtained from the quenched samples sheared at the highest $\sigma_{w}(65 \mathrm{kPa})$ for both $t_{s}=7$ and $12 \mathrm{~s}$. For optical microscopy with polarized light, specimens $10-20 \mu \mathrm{m}$ thick were examined with crossed polarizers oriented at $\pm 45^{\circ}$ from the flow direction. For microfocus WAXD characterization, samples between 0.5 and $1 \mathrm{~mm}$ were examined using the ID13 beamline of the European Synchrotron Radiation Source (Grenoble, France). The x-ray wavelength was $0.984 \AA$. A beam of $5 \mu \mathrm{m}$ size was used to scan the sample in $5 \mu \mathrm{m}$ steps, and the WAXD patterns were collected with a MARCCD camera of $1024 \times 1024$ pixels, and pixel size of $157.88 \mu \mathrm{m}$. The x-ray beam for these ex-situ measurements was directed along the vorticity direction (probing structure in the $x y$ plane, Fig. 2), orthogonal to the orientation of the in-situ optical and x-ray beams (Fig. 2). Rastering the micro-beam across the sample provided spatially resolved WAXD patterns for the final structure as a function of distance from the wall, i.e., as a function of shear stress. To correlate the 

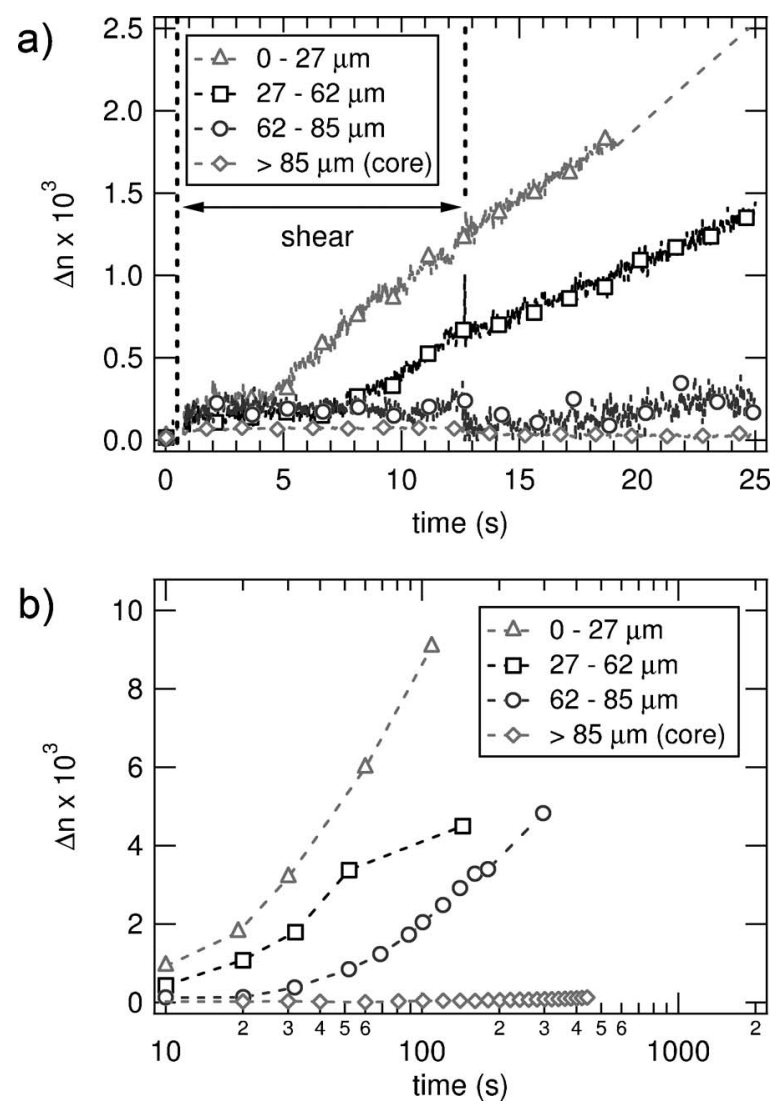

FIG. 5. (a) Average birefringence for each section during and immediately after a $12 \mathrm{~s}$ shear pulse. (b) Development of the average birefringence for each depth section after shearing for $12 \mathrm{~s}$ at $137{ }^{\circ} \mathrm{C}$. Depths indicated are with respect to $\sigma_{w}=65 \mathrm{kPa}$.

results obtained from depth sectioning of the real-time WAXD patterns, we show the average of the ex-situ patterns that fall within each of the slices examined using real-time depth sectioning.

\section{RESULTS}

The depth dependence of the real-time retardance and WAXD in a sample subjected to a wall shear stress of $65 \mathrm{kPa}$ are examined first for $t_{s}=12 \mathrm{~s}$ and then compared to those for $t_{s}=7 \mathrm{~s}$. Afterward, we relate the real-time depth sectioning data to the final structure observed ex-situ using microfocus WAXD. The depth sectioning results are visualized in terms of different distances from the wall for the sample sheared at the highest stress; alternatively, they may be interpreted in terms of the local shear stress to make contact with theoretical models of flow-induced structure formation.

\section{A. Depth-resolved structure development for $t_{s}=12 \mathrm{~s}$}

At very early times - during and right after shearing - the sensitivity and speed of rheo-optical measurements reveal the formation of oriented precursors and the initial growth of crystals on them via the average birefringence of each depth slice [Fig. 5(a)]. Upon the inception of shear, the melt stress optical contribution appears immediately as 
a small step increase. For the outer section (up to $27 \mu \mathrm{m}$ from the wall), a further increase in birefringence-beyond the melt contribution-starts at $t^{*} \sim 4.5 \mathrm{~s}$ after the inception of flow. A similar increase in birefringence beyond that due to the melt occurs in the adjacent section, 27-62 $\mu \mathrm{m}$ from the wall, but it starts later (after $7 \mathrm{~s}$ of shearing) and grows more slowly [Fig. 5(a)]. The birefringent structure formed in the outermost layers appears not to relax upon the cessation of flow (no drop in $\Delta n$ upon cessation of flow). Prior measurements (averaged over the entire thickness of the sample) have consistently shown an abrupt drop in retardance upon the cessation of flow [Kumaraswamy et al. (1999a)], which is also evident in the present experiment. The new and significant information obtained by depth sectioning is that the outer layer, which contributes substantially to the birefringence, does not appreciably contribute to the relaxation process observed after cessation of flow.

After cessation of $12 \mathrm{~s}$ of flow, the birefringence of the outermost $27 \mu \mathrm{m}$ continues to increase rapidly [Fig. 5(a)], as fast as it did during shear for $t>t^{*}$. In the adjacent depth section (27-62 $\mu \mathrm{m}$ from the wall), the rate of increase of $\Delta n$ diminishes when flow stops. In the "dense sausage layer" [Fernandez-Ballester (2007)] ( 60-100 $\mu \mathrm{m}$ observed ex-situ [Fig. 3(a)], left micrograph), the retardance develops gradually and only becomes evident after the cessation of flow [Fig. 5(b)]. At still greater depth, birefringence remains very low.

Growth of the parent (110) area $A_{P, 110}$ [Fig. 6(a)] becomes measurable several seconds after cessation of flow. Like $\Delta n(t)$ [Fig. 5(b)], the population of parent crystallites increases fastest for the highest shear stress and progressively slower for the inner sections. The core section $(>102 \mu \mathrm{m})$ shows very little growth for the duration of the experiment $(\sim 1200 \mathrm{~s})$. The increase of birefringence after the cessation of flow and the increase in the population of oriented parent crystallites both arise from the growth of kebabs, which serve as a reporter of the underlying oriented precursors (shish) created during flow [Kumaraswamy et al. (2000); Fernandez-Ballester et al. (2008)]. The earliest measurable growth of oriented crystallites from them is most closely related to the precursor population created during flow. Section V will examine the effects of stress on the creation of precursors evident in the observed initial growth rates of $A_{P, 110}$ in each depth section.

In the two outermost sections, the rapid initial rise in $A_{P, 110}$ is followed by an abrupt decrease in growth rate within $100 \mathrm{~s}$ of the cessation of shear [Fig. 6(a)]. In contrast, the depth slice corresponding to the sausage region (third slice of $t_{s}=12 \mathrm{~s}$ ) exhibits a much more gradual change in the rate of the growth in $A_{P, 110}$. The decrease in growth rate is attributed to the impingement of cyllindrulites [Kumaraswamy et al. (2000); Kornfield et al. (2002)]. Given that the lamellae are growing outward under isothermal conditions into a relaxed melt, their linear growth velocity is the same in all of these experiments and the time to impingement is governed by the separation distance between precursors. Therefore, Sec. V will examine the density of precursors (thread length per volume) using both the initial growth rate (above) and the impingement time.

As seen in Fig. 3(c), crystallites formed within $1200 \mathrm{~s}$ of the shear pulse in the three outer sections are very highly oriented [Fig. 6(b)]; the FWHMs corresponding to the two higher shear stresses are very narrow and indistinguishable. In contrast, the core section presents very weak orientation (greater FWHM with large uncertainty due to weak poorly oriented peaks on a strong amorphous halo [Fig. 3(c), right]).

The ratio of parent $(P)$ lamellae to daughter $(D)$ lamellae is greatest for the outermost $35 \mu \mathrm{m}$, intermediate for the second slice, and least for the two inner sections [Fig. 6(c)]. Note that daughter crystallites are nearly orthogonal to the parents and tend to cancel out the birefringence contribution of the parent population. Therefore, the WAXD results provide insight into the trends seen in the birefringence [Kumaraswamy et al. (2000); 


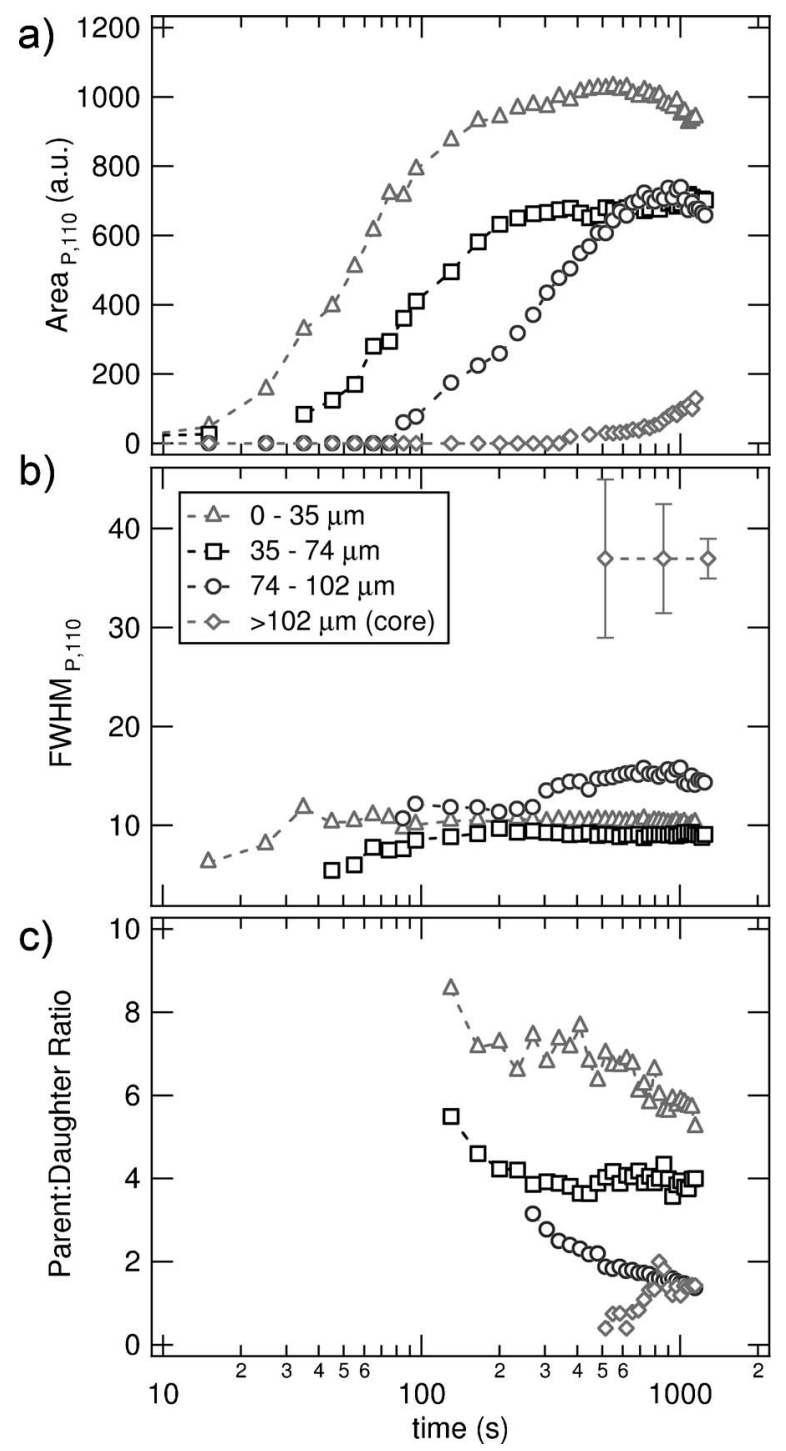

FIG. 6. Transient WAXD results based on the (110) reflection in the four depth sections examined for $12 \mathrm{~s}$ and $T_{x}=137^{\circ} \mathrm{C}$. (a) Area of the parent 110 peaks. (b) FWHM of the parent 110 peak calculated after fitting the peaks in the 110 azimuthal scan. (c) Ratio of parent-to-daughter lamellae after performing a geometrical correction of the observed intensity. Lines connect the data points; symbol shapes distinguish the depth sections indicated with respect to $\sigma_{w}=65 \mathrm{kPa}$.

Kumaraswamy et al. (2004)]. The slower increase in retardance in the second depth section relative to the first is due in part to a slower growth of the parent population (which correlates with a lower concentration of thread-like precursors) and in part due to the greater population of daughter crystallites that grows with them. In addition, the absence of birefringence in the central section does not exclude the formation of a small oriented population. The large ratio of daughters to parents in the weakly oriented population evident in the core WAXD pattern appears to be responsible for the lack of birefringence. 


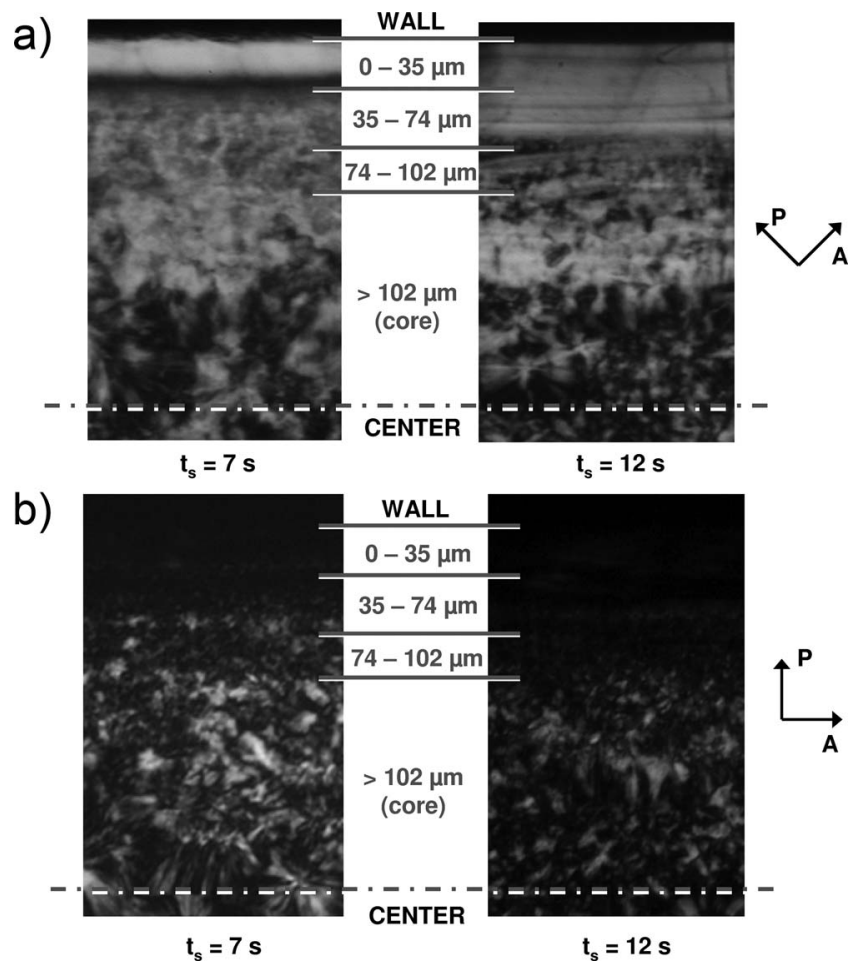

FIG. 7. OPM of samples sheared using the highest wall shear stress $\left(\sigma_{w}=65 \mathrm{kPa}\right)$ for 7 and $12 \mathrm{~s}$ at $137{ }^{\circ} \mathrm{C}$ with polarizer and analyzer (a) at $\pm 45^{\circ}$ and (b) at $0^{\circ}$ and $90^{\circ}$ with respect to the flow direction (horizontal). The center of the sample is indicated by the dashed-dotted line.

\section{B. Effect of shearing time $\left(t_{s}=7 \mathrm{~s}\right.$ vs $\left.12 \mathrm{~s}\right)$}

Ex-situ polarized optical micrographs of samples sheared at $\sigma_{w}=65 \mathrm{kPa}$ (Fig. 7) show that the highly oriented skin is much thinner for the sample sheared for $t_{s}=7 \mathrm{~s}$ $\left(\sim 30 \mu \mathrm{m}\right.$, first slice) than for the case of $t_{s}=12 \mathrm{~s}(\sim 60 \mu \mathrm{m}$, first depth section and most of the second). From the real-time WAXD results for $t_{s}=12 \mathrm{~s}$ (above) and for $t_{s}$ $=7 \mathrm{~s}$ (below), it is clear that most of the crystallites formed at depths $>102 \mu \mathrm{m}$ did not form during the first $1200 \mathrm{~s}$. The structures seen ex situ in the core of these samples (which were cooled from $T_{x}$ to $25^{\circ} \mathrm{C}$ after $5400 \mathrm{~s}$ ) grew non-isothermally after the flow cartridge was removed for cooling [Kumaraswamy et al. (1999b)]. These inner depths include two morphologies for both $t_{s}=7$ and $12 \mathrm{~s}$ : a weakly oriented region from the oriented skin to $\sim 175 \mu \mathrm{m}$ from the wall (third section and half of the fourth) with sausage-like birefringent structures [Fernandez-Ballester (2007)] and an unoriented inner core $(>175-200 \mu \mathrm{m})$.

Structure development during flow in the outer section $(0-27 \mu \mathrm{m})$ is very similar for both $t_{s}=7$ and $12 \mathrm{~s}$ [Fig. 8(a)]. This is expected for the first $7 \mathrm{~s}$ of the $12 \mathrm{~s}$ shear pulse (the melt birefringence up to $t=4.5 \mathrm{~s}$ and the subsequent upturn), indicating reproducibility of the experiments and the depth sectioning analysis. Surprisingly, between 7 and $12 \mathrm{~s}$, the increase in birefringence following the cessation of shear for $t_{s}=7 \mathrm{~s}$ also coincides with that observed during continued shearing for the case $t_{s}=12 \mathrm{~s}$ : at all longer times, the increase in birefringence for $t_{s}=7 \mathrm{~s}$ tracks that of $t_{s}=12 \mathrm{~s}$ [Fig. 8(b)]. The additional $5 \mathrm{~s}$ of shear for $t_{s}=12 \mathrm{~s}$ appear to have had no consequence at the highest stress. Analo- 

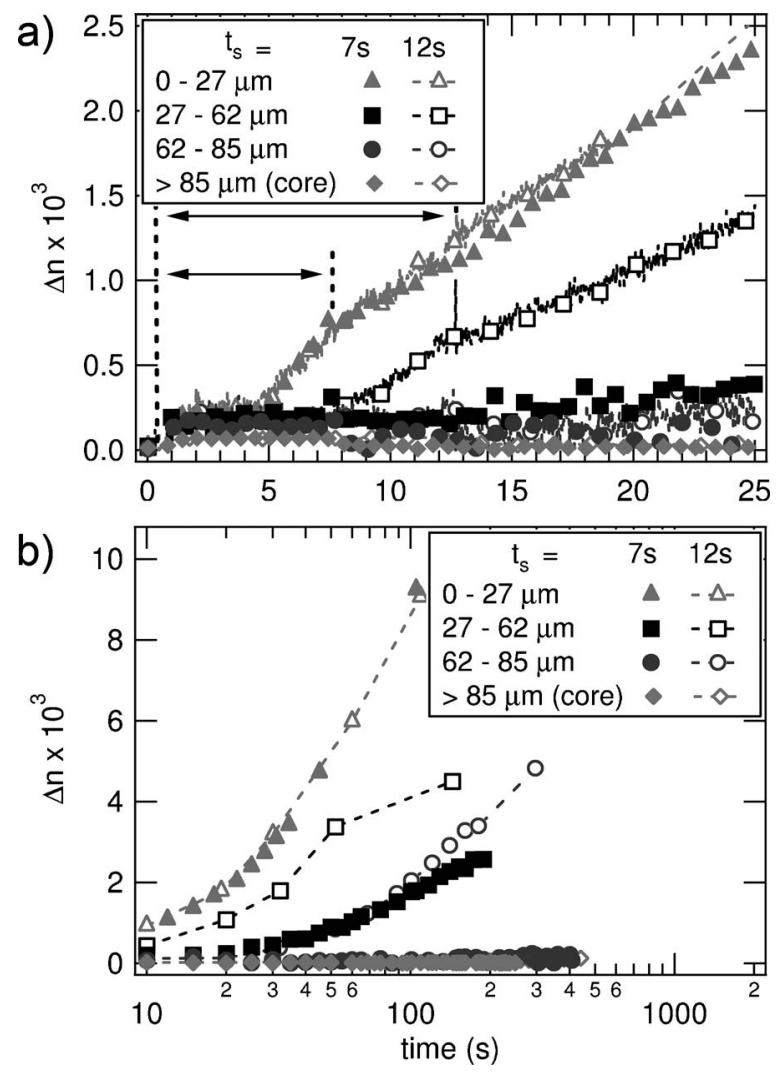

FIG. 8. (a) Transient average birefringence for $t_{s}=7 \mathrm{~s}$ and $t_{s}=12 \mathrm{~s}$ in each section during and immediately after flow. (b) Average birefringence at later times for each depth section for $t_{s}=7 \mathrm{~s}$ and $t_{s}=12 \mathrm{~s}$. To compare the behavior following $7 \mathrm{~s}$ of shear to that after $12 \mathrm{~s}$, the earlier results for $t_{s}=12 \mathrm{~s}$ in Fig. 5 are shown as open symbols, while the results for $7 \mathrm{~s}$ as depicted as filled symbols. Depths indicated are with respect to $\sigma_{w}$ $=65 \mathrm{kPa}$.

gously, the initial growth rate of the $A_{P, 110}$ in the outer depth slice after the cessation of flow is very similar for $t_{s}=7$ and $12 \mathrm{~s}$ [Fig. 9(a)]. The roll-off and plateau of $A_{P, 110}$ of the outermost slice (up to $35 \mu \mathrm{m}$ ) are also much the same for $t_{s}=7 \mathrm{~s}$ and $t_{s}=12 \mathrm{~s}$ [Fig. 9(a), filled vs open triangles]. Likewise, the parent 110 peaks reveal a high degree of orientation (FWHM) which is indistinguishable between $t_{s}=7$ and $12 \mathrm{~s}$ [Fig. 9(b)]. The only manifest difference is the higher $P: D$ ratio for $t_{s}=12 \mathrm{~s}(P: D \sim 6)$ when compared to $t_{s}=7 \mathrm{~s}(P: D \sim 3)[$ Fig. 9(c)].

Inner depths in the sample confirm agreement between the transient birefringence during the $t_{s}=7 \mathrm{~s}$ pulse and the first $7 \mathrm{~s}$ of the $t_{s}=12 \mathrm{~s}$ shear pulse [Fig. 8(a)]; for this short shearing time, all inner sections show only melt birefringence (no upturn). In contrast to the outermost section, the effects of shearing time are strong in the second and third depth sections. After cessation of $7 \mathrm{~s}$ of flow, little birefringence remains [27-62 and 62-85 $\mu \mathrm{m}$ slices, Fig. 8(a)] and, accordingly, the development of oriented structures is much weaker than after the $t_{s}=12 \mathrm{~s}$ pulse [filled vs. open squares and circles in Fig. 8(b)]. WAXD shows that the negligible $\Delta n$ of the third section [filled circles in Fig. 8(b)] does not mean crystallization is isotropic; instead the absence of optical anisotropy is due to a combination of slower growth of $A_{\mathrm{P}, 110}$ [Fig. 9(a)], weaker alignment of the crystallites that form [Fig. 9(b)], and an increase in the proportion of daughter crystallites [Fig. 

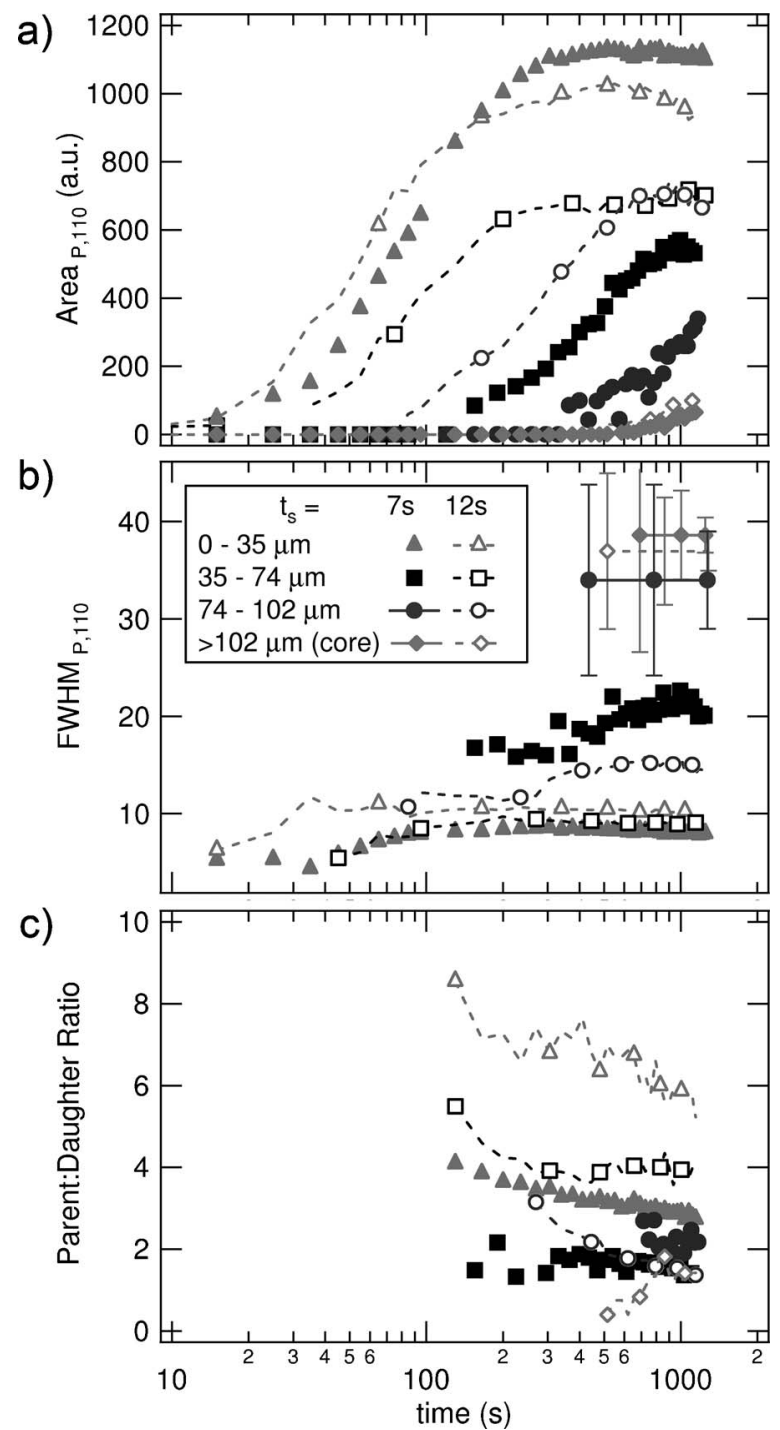

FIG. 9. Comparison of WAXD depth sectioning results for $t_{s}=7$ and $12 \mathrm{~s}$ at $137{ }^{\circ} \mathrm{C}$. The earlier results for $t_{s}=12 \mathrm{~s}$ in Fig. 6 are shown as open symbols together with the results for $t_{s}=7 \mathrm{~s}$ as filled symbols. (a) Area of parent 110 peak. (b) FWHM of parent 110 peak. (c) Parent-to-daughter ratio after geometric correction.

$9(\mathrm{c})]$ in $t_{s}=7 \mathrm{~s}$ relative to $t_{s}=12 \mathrm{~s}$. In the core of the sample, negligible oriented growth occurs for $t_{s}=7 \mathrm{~s}$, indicated by the combination of $\Delta n$ and $A_{P, 110}$ both remaining negligible. Note that both $\Delta n$ and WAXD results show that, although increasing shearing time from 7 to $12 \mathrm{~s}$ has little effect at the highest and lowest stresses, it has pronounced effects at intermediate stresses. This will prove important in Sec. V.

We now examine the relationship between the structure revealed by the depthsectioned real-time WAXD patterns (at $1145 \mathrm{~s}$, the end of the $137^{\circ} \mathrm{C}$ isothermal experiment) and the ex-situ spatially resolved WAXD patterns obtained after the sample was cooled to room temperature and stored for about six months (Fig. 10). Comparison of ex-situ scans with the last real-time patterns prior to cooling indicates that the regions densely populated with oriented precursors have a morphology that is already determined 
$7 \mathrm{~s}$
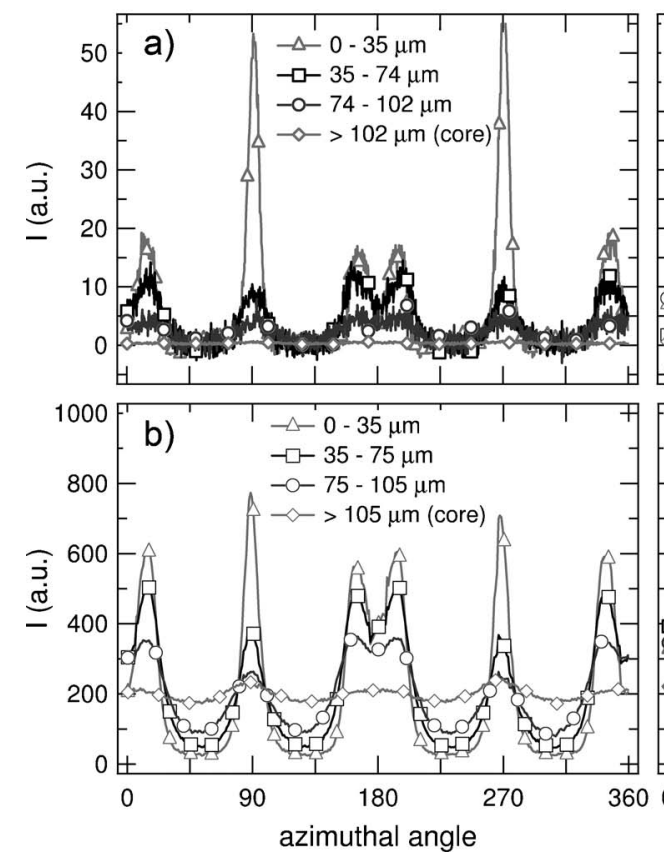

$12 \mathrm{~s}$
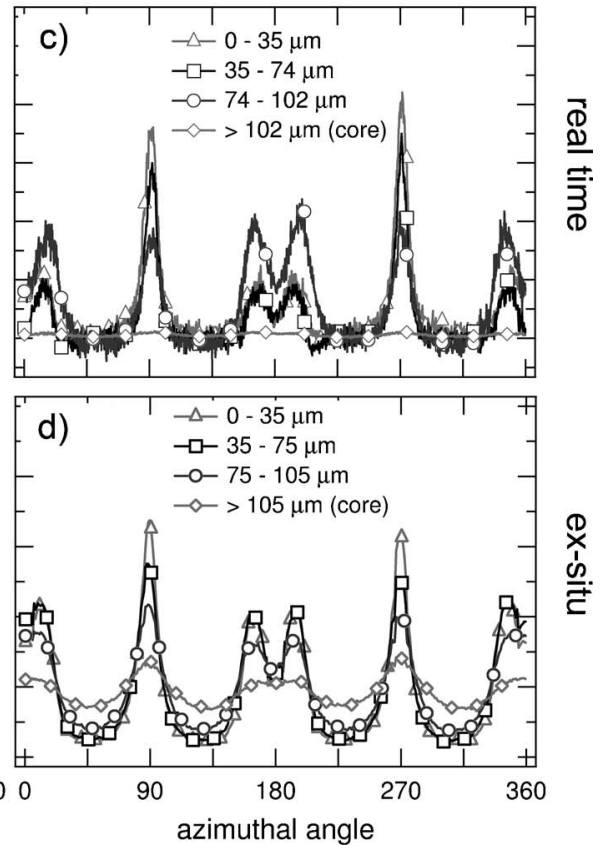

FIG. 10. Azimuthal scan of the (110) reflection of [(a) and (c)] real-time patterns with beam propagating in the $y$ direction after applying depth sectioning and [(b) and (d)] microfocus ex-situ patterns with beam propagating along the $z$ direction for specimen sheared for $t_{s}=7 \mathrm{~s}\left[(\mathrm{a})\right.$ and (b)] and for $t_{s}=12 \mathrm{~s}[(\mathrm{c})$ and (d)]. The real-time WAXD profiles have had the amorphous contribution subtracted, while the ex-situ micro-diffraction pattern includes the remaining amorphous halo present in the fully solidified state. The azimuthal scans for the core in parts (a) and (c) have distinct peaks for the parent population and doublets for the daughters [Fig. 3(c)] that are not visible here due to their low intensity relative to the other sections.

during the isothermal crystallization and that, at the opposite extreme, the crystallization of the central section of the sample $(>102 \mu \mathrm{m})$ occurs mainly after sample removal and cooling. The highly oriented layers $\left(0-35 \mu \mathrm{m}\right.$ for $t_{s}=7 \mathrm{~s}$, and both $0-35$ and $35-74 \mu \mathrm{m}$ for $t_{s}=12 \mathrm{~s}$ ) show that the degree of orientation present in situ at $1145 \mathrm{~s}$ is nearly retained by the crystallites that form during cooling. The absence of oriented precursors in the core section $(<102 \mu \mathrm{m})$ was manifested by negligible diffraction at $1145 \mathrm{~s}$ and a nearly isotropic distribution of crystallites evident in the ex-situ pattern. Interestingly, for $t_{s}=12 \mathrm{~s}$, the $e x$-situ central section shows that the predominantly unoriented crystallites are accompanied by a small mildly oriented population (which is barely evident in the equivalent section in the ex-situ sample for $t_{s}=7 \mathrm{~s}$ ). In the moderately oriented sections (35-75 and 75-105 $\mu \mathrm{m}$ for $t_{s}=7 \mathrm{~s}$ and 75-105 $\mu \mathrm{m} t_{s}=12 \mathrm{~s}$ ), the oriented crystallites (parent peaks at $90^{\circ}$ and $270^{\circ}$ and daughter doublets) present in-situ at $1145 \mathrm{~s}$ increased during cooling and aging. In addition, an appreciable unoriented contribution in the final crystalline morphology formed during cooling and aging (minima between peaks offset from zero in ex-situ patterns but not in the in-situ patterns).

\section{DISCUSSION}

Shear-induced structure is relevant to diverse materials: semicrystalline polymers [Eder et al. (1990); Keller and Kolnaar (1997); Kumaraswamy (2005); Somani et al. (2005); Baert et al. (2006); Hassell and Mackley (2008)], block copolymers [Chen et al. 
(1997); Krishnan et al. (2005)], wormlike micelles [Berret et al. (1997); Becu et al. (2004); Lee et al. (2005)], and liquid-crystalline polymers [Rendon et al. (2007)], to name a few. Measurements of transient optical anisotropy [Hongladarom et al. (1993); Fuller (1997)], small-angle light scattering [Norman et al. (2006)], SAXS [Caputo et al. (2002)], and WAXD [Somani et al. (2001); Kumaraswamy et al. (2004)] are important tools for understanding microstructure evolution during flow. To facilitate the interpretation of these observables, measurements are often performed using rheometric flows that impose uniform deformation throughout the sample [Somani et al. (2006); Lagasse and Maxwell (1976); Nogales et al. (2001); Baert et al. (2006)]. Alternatively, pressuredriven flow offers the advantage of readily controlled-stress conditions and the ease of sudden inception and cessation of shear. The depth sectioning method allows local information (corresponding to nearly uniform conditions) about material structure to be obtained from pressure-driven flow through a rectangular slit.

To illustrate how the real-time depth sectioning method is used to analyze the effect of shear on structure development, we examine polymer crystallization as a function of shear stress (which, for a given maximum wall shear stress, can be visualized in terms of distance from the channel wall, i.e., depth within the sample). In contrast to "postmortem" methods that probe the depth dependence of the final structure, the present method provides information on how the structure forms. The kinetics of flow-induced crystallization and morphology development provides insight into the process of creation of highly oriented precursors during flow (which depends strongly on the stress imposed) and their impact on the ensuing crystallization.

The four most important new findings that have been enabled by depth sectioning are (1) in the highly oriented regions, negligible decrease in birefringence occurs upon cessation of shear, (2) the transition in growth kinetics in the oriented skin changes abruptly upon impingement of the cyllindrulites, (3) after shish have saturated, further shearing hardly changes the rate or morphology of crystallization on them, and (4) before shish have saturated, the increase in thread-length per volume between two shearing times can be estimated from the depth-resolved data. The first two of these are readily evident in the results of depth sectioning applied to the experiment with $12 \mathrm{~s}$ of shearing discussed next. The third and fourth are exposed by comparing depth sectioning results for two different shearing times (7 and $12 \mathrm{~s}$ ) and open the way for future work to determine the kinetic parameters for the propagation of shish as functions of molecular attributes (such as molar mass distribution) and flow conditions (including temperature and stress).

\section{A. Shish-kebabs do not relax upon cessation of shear}

Using the depth sectioning method to isolate the signal corresponding to the highly oriented skin clearly confirmed that only these sections exhibit the rheo-optical signature of the formation of oriented precursors - an upturn in birefringence during the shear pulse [Fig. 5(a)]. Previous studies that compared the averaged signal through the flow channel and the ex-situ morphology noted that an upturn in birefringence during shear and the subsequent rapid growth of retardance after the cessation of flow correlate with the ex-situ observation of a highly oriented skin [Kumaraswamy et al. (1999a); Langouche (2006)]. Here, by applying depth sectioning, we are able to directly ascribe the upturn in birefringence to the depth sections that later show the oriented skin in the ex-situ micrographs.

The observation that the birefringence that has built up in the sections corresponding to highly oriented skin does not relax when the flow is stopped [Fig. 5(a)] indicates that the oriented precursors and associated oriented lamellae developed during shear do not 


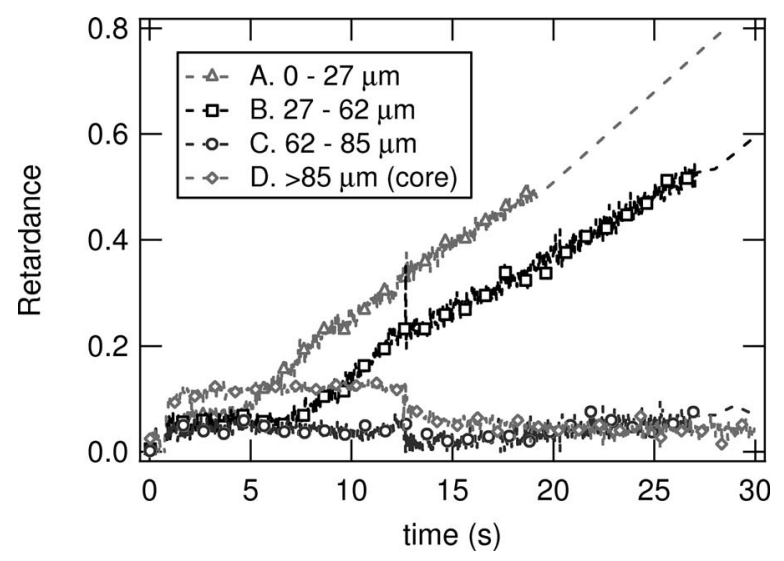

FIG. 11. Retardance contribution of each depth section for $12 \mathrm{~s}$ shearing time at $137{ }^{\circ} \mathrm{C}$. These data were used to compute the average birefringence depicted in Fig. 5(a) using the thickness of each depth slice.

noticeably decay at this temperature $\left(137^{\circ} \mathrm{C}\right)$, in accord with independent findings using a sliding plate to impose short-term shear [Fig. 3 of Langouche (2006)]. Therefore, the partial drop in $I_{\perp} /\left(I_{\perp}+I_{\|}\right)$observed in the averaged signal through the entire channel [e.g., Fig. 6 of Kumaraswamy et al. (1999a), and inset of Fig. 6 of Kumaraswamy et al. (1999b)] corresponds mainly to the relaxation of the melt in the central parts of the sample. To confirm this interpretation, we examine the total retardance of each depth section (Fig. 11), i.e., the average birefringence of a section multiplied by its thickness. Indeed, the central sections, in which the birefringence during flow is solely due to the melt stress-optical behavior, fully account for the relaxation of retardance upon cessation of flow that is observed in the depth-average measurements here, and likely in previously reported experiments.

\section{B. Abrupt transition in growth kinetics in highly oriented skin at impingement}

The change in growth rate in $A_{P, 110}$ observed in the outer depth sections is attributed to the impingement between kebabs propagating out from neighboring threads. Prior investigations of real-time WAXD [Kumaraswamy et al. (2000); Kornfield et al. (2002)] reported a rapid growth of highly oriented WAXD followed by the saturation of the growth of crystalline peaks and hypothesized that this transient corresponded to the growth of highly oriented crystallites on thread-like precursors until lamellae growing off neighboring threads impinged with one another.

Application of depth sectioning allows us to compare the relative distance between threads and the regularity of that interval at different depths from the wall. After the cessation of flow, under quiescent conditions, kebabs grow radially outward from the shish that were created during the shear. Since the temperature is the same for all the depth sections, the quiescent linear growth rate of the oriented lamellae is the same at any depth. Therefore, the time at which lamellae growing off neighboring shish impinge depends only on the distance between the threads, while the sharpness of the transition gives an indication of the homogeneity of the spacing. If no depth sectioning is applied, the average signal reflects successive growth rate transitions, precluding comparisons between different applied shear stresses (i.e., distances from the wall). 
The time at which the "knee" in growth rate occurs for the two sections corresponding to the highly oriented skin $\left(t_{s}=12 \mathrm{~s}\right)$ indicates that the separation between the shish is small $(\leq 1 \mu \mathrm{m}){ }^{2}$ A high density of threads in the oriented skin is consistent with the very high degree of crystallite orientation in these depth sections: the threads template highly oriented growth and the lamellae impinge before they diverge. The sharpness of the change in growth rate suggests that the spacing between shish in each section is fairly uniform.

Farther from the wall, the knee is later and broader for the sausage region (third slice of $t_{s}=12 \mathrm{~s}$ ), indicating that few thread-like precursors had formed during flow and that they have large and inhomogeneous inter-thread spacings-as confirmed by polarized optical microscopy (OPM). The shish formed were so scarce that they did not cause an upturn in the birefringence during flow. The lower crystallite orientation measured in the sausage area is also consistent with a lower density of threads. The crystalline lamellae nucleated on the threads propagate to much larger radius in the sausage region than in the skin, allowing non-crystallographic branching to decrease the orientation correlation of the direction of crystal growth.

\section{Saturation of shish}

At the highest stresses (outermost depth section, from the wall to $35 \mu \mathrm{m}$ ), both $t_{s}$ $=7$ and $12 \mathrm{~s}$ induce a highly oriented skin and the development of the real-time observables is very similar. From the moment shear is started up to $7 \mathrm{~s}$ of shear, the rise of the birefringence is the same for both experiments of $t_{s}=7$ and $12 \mathrm{~s}$, as expected [Fig. 8(a)]. However, it is remarkable that for the next $5 \mathrm{~s}$ (that is, after the cessation of flow for $t_{s}=7 \mathrm{~s}$ but while the shear pulse is still imposed on the melt for $t_{s}=12 \mathrm{~s}$ ), the growth of birefringence is still very similar. In other words, the additional $5 \mathrm{~s}$ of shear in the case of $t_{s}=12 \mathrm{~s}$ have no additional effect when compared with the case in which flow has stopped after $7 \mathrm{~s}$. The saturation of the shish is also manifested after the cessation of shear by the similar growth rate of kebabs from them: the resemblance of the initial growth rate of the $A_{P, 110}$ peaks for the outermost layer is very similar for $t_{s}=7 \mathrm{~s}$ and $t_{s}=12 \mathrm{~s}$ (Fig. 9). The similarly sharp and early transition in the growth rate of the kebabs, measured in $A_{P, 110}$, suggests that the inter-shish spacing was already established during the first $7 \mathrm{~s}$ of shearing. Depth sectioning enabled this discovery. In the depth-averaged observables, the contribution of inner sections to the overall measured signal (in this case, the signal corresponding to the second depth slice of $t_{s}=12 \mathrm{~s}$ ) masks the saturation behavior observed in the outer section.

Saturation of the density of threads has been previously observed [Kornfield et al. (2002)]. In that study, increasing the shearing time from 4 to $8 \mathrm{~s}$ did not enhance the density of threads in the oriented skin as observed by transmission electron miscropy. A number of possible explanations of saturation can be put forward. One of them invokes the depletion of long chains (which are known to be crucial for the development of threads) at a critical thread density. If there are many threads very close together, most long chains may already be involved in them and further propagation cannot occur. However, Kimata and co-workers recently discovered that long chains are not overrepresented in the thread-like precursors relative to the rest of the material [Kimata et al. (2007)]. Therefore, long chains are not depleted by the formation of shish. An alternate explanation may be that when the thread-length/volume is very high, the incipient shishkebabs may interact and hinder each other from elongating further (see below).

\footnotetext{
${ }^{2}$ The linear growth rate at $137{ }^{\circ} \mathrm{C}$ was extrapolated from literature as $G \sim 0.003 \mu \mathrm{m} / \mathrm{s}$ [Janimak et al. (1991)].
} 
It is interesting to note that the rate of growth of birefringence upon the cessation of 7 s of shear hardly changes when the flow is stopped [Fig. 8(a)]. This suggests that the physics dominating the growth of oriented crystallites during shear had already changed from a mechanism highly sensitive to flow to a mechanism that is independent of flow. Specifically, when saturation is reached, the deformation of the highly oriented skin may have slowed or ceased. Perhaps the first lamellae to nucleate on a given thread have already advanced a few hundred nanometers and these "catch" on each other as shish translate along nearby streamlines. Plausibility of this scenario is indicated by the significant population of oriented crystallites already formed within $7 \mathrm{~s}$ of shear: kebabs already pervade approximately $6 \%$ of the volume of the oriented skin after just $7 \mathrm{~s}$ of shear, based on the ratios of the birefringence at $7 \mathrm{~s}$ to that at $80 \mathrm{~s}$ (Fig. 8), together with the ratio of $A_{P, 110}$ at $80 \mathrm{~s}$ to that well after impingement [ 300 s (Fig. 9)].

Although the additional $5 \mathrm{~s}$ of shearing between $t_{s}=7$ and $12 \mathrm{~s}$ hardly changes threadlength, it does affect the subsequent $P: D$ ratio in the outermost $35 \mu \mathrm{m}$. This is surprising, since most crystal growth of both parents and daughters occurs after the cessation of flow (i.e., under quiescent isothermal conditions such that $P: D$ is expected to be the same). A previous study using depth-averaged observables also found a greater $P: D$ ratio arising from row nucleation when longer shearing times at high shear stresses were imposed on a iPP melt [Kumaraswamy et al. (2004)]. The parent-to-daughter ratio is known to be a function of temperature, but here conditions are isothermal. Kumaraswamy et al. (2004) explained the increase in $P: D$ in terms of the preferential growth of the parent lamellae (which have the chain axis along the flow direction) formed during shear. In their case, a substantial amount of the kebab crystallization had occurred during flow (about 50\% relative to the crystallinity at impingement), so a greater $P: D$ ratio during flow would skew the final value of $P: D$ even if the growth after the cessation of shear occurred with the ratio typical of quiescent conditions. However, the present data demand a different explanation: comparatively less growth occurred by the end of the shear pulse. For the outermost section [Fig. 6(a)], $A_{P, 110}$ is $\sim 50$ a.u. $3 \mathrm{~s}$ after the cessation of flow, while at the time of impingement $(t \sim 90 \mathrm{~s}) A_{P, 110}$ has reached a value 15 times higher. Therefore, the structures created by $12 \mathrm{~s}$ of shear must differ from those created by $7 \mathrm{~s}$ of shear in a way that influences the $P: D$ ratio of the quiescent growth that occurs long after the cessation of shear.

\section{Propagation of shear-induced precursors}

For the current experiments, the growth of kebabs after the cessation of shear occurs under quiescent isothermal conditions, i.e., the linear growth rate of the kebabs is fixed. Therefore, at early times before impingement occurs, the growth rate of the oriented lamellae on the shish serves as a reporter of the total length of flow-induced precursors that have formed. (At later times, impingement causes a negative deviation of growth rates relative to linear proportionality to the total length of shish.) Consequently, the rate of formation of shish at different levels of stress between 7 and $12 \mathrm{~s}$ of shearing can be deduced by comparing the initial growth of $A_{P, 110}$ and birefringence after the cessation of flow.

To compare growth rates of $\Delta n$ and $A_{P, 110}$ to each other, the initial slopes of $\Delta n$ [Fig. 8(b)] and $A_{P, 110}$ [Fig. 9(a)] after the cessation of the shear pulse were determined and normalized by the slope observed for the greatest stress $(65 \mathrm{kPa}$ for $\Delta n$ and $64 \mathrm{kPa}$ for $\left.A_{P, 110}\right)$ and longest shearing time $\left(t_{s}=12 \mathrm{~s}\right)$. The normalized initial rates of increase of $\Delta n$ and $A_{P, 110}$ vary similarly with the local stress (Fig. 12). Given that the daughter lamellae negate some of the birefringence contributed by the parent lamellae, the close 


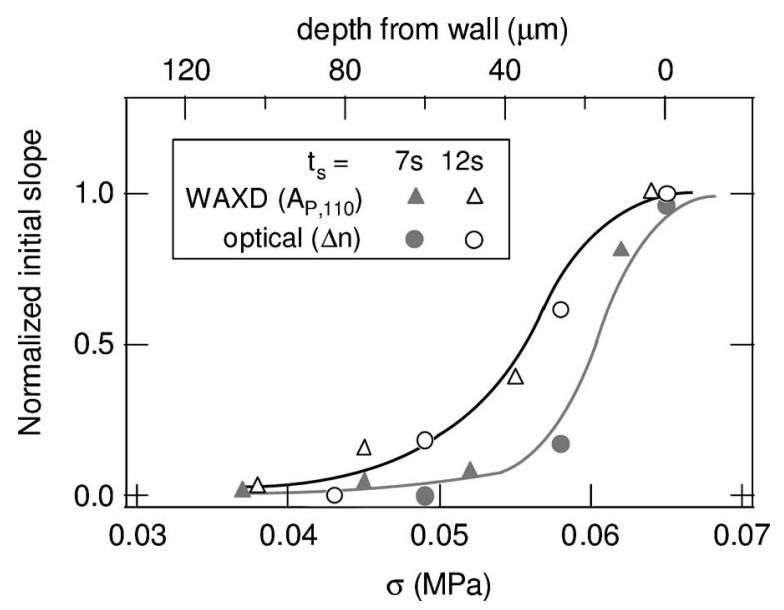

FIG. 12. Dependence of initial rate of growth of normalized average birefringence and $A_{P, 110}$ on shear stress for $t_{s}=7$ and $12 \mathrm{~s}$. The distance from the wall indicated on the top axis is based on a sample sheared at the highest wall shear stress, corresponding to the micrographs shown in Fig. 7.

agreement between the length of precursors deduced from the rheo-optical and the rheo$\mathrm{X}$-ray data suggests that the amount of oriented precursors formed can be estimated from birefringence measurements, particularly for the highly oriented sections. X-rays are necessary to obtain further information about the detailed morphology of the shish-kebab structure.

At intermediate stresses $(\sigma \sim 48-60 \mathrm{kPa})$, early growth rates for $12 \mathrm{~s}$ are significantly higher than those of $7 \mathrm{~s}$, indicating that the propagation of threads continued during the additional $5 \mathrm{~s}$ of shear. In contrast, at low and high shear stresses, the initial slopes are similar. At the lowest stress, no thread formation occurs, even during $12 \mathrm{~s}$ of shear, while at the highest stress saturation of threads has occurred so that no additional thread-length is created from $t_{s}=7$ to $12 \mathrm{~s}$. Therefore, the comparison of different shearing times for intermediate flow conditions (at high enough stress to induce threads, but prior to saturation) provides a promising approach to compare the velocity of propagation of oriented precursors as a function of flow and thermal conditions.

\section{CONCLUSIONS}

We have illustrated "real-time depth sectioning" by applying it to the flow-induced crystallization of isotactic polypropylene. This method combines the advantages of pressure-driven flow (e.g., the ease of sudden inception and cessation of shear, the unambiguous control over the shear stresses imposed on the melt, and the ability to apply large shear stresses that are relevant to the flow-induced structures induced during injection molding and extrusion) with the key advantage of rheometric flows (observing structure evolution at a specific stress).

The creation of thread-like precursors depends strongly on stress. Therefore, the depth sectioning method exposes the effect of one of the most important variables impacting flow-induced crystallization. The in-situ measurements are consistent with the depth dependence of the final morphology; the real-time observations clarify the underlying basis of structure development at different levels of stress. Highly oriented precursors initiate first in the regions of greatest shear stress (near the wall). For the highest stress used (65 $\mathrm{kPa}$ ), we observed that structure development during and after flow saturated within the 
first $7 \mathrm{~s}$ of shearing, so that the kinetics of subsequent crystallization was very similar for both $t_{s}=7$ and $12 \mathrm{~s}$. Also we deduced that at $137^{\circ} \mathrm{C}$, significant kebab overgrowth on the threads occurs during flow and that these precursors (plus overgrowth) do not relax after cessation of flow. At lower stresses, thread-like precursors formed later and their density (thread length per volume) increased at a slower rate with shearing time. The ability to isolate events at different levels of stress opens the way for studying how the creation of oriented thread-like precursors varies with other parameters as well, such as shearing time (as shown here), material properties (molecular weight, molecular weight distribution, isotacticity), and temperatures of shear and/or crystallization.

This approach is applicable to diverse complex fluids, including block copolymers, polymer blends, and colloidal suspensions.

\section{ACKNOWLEDGMENTS}

Financial support for this work was provided by the National Science Foundation under Grant Nos. DMR-0505393 and DMI-0218112, and by the ARCS Foundation. We are grateful to Dr. Weijun Zhou and Dr. R. L. Sammler of Dow Chemical Co. for kindly providing the materials used in this study. We thank Jan Willem Housmans for microfocus measurements, and Lixia Rong and Igors Sics for help with experimental setup at X27C (NSLS). We acknowledge NSLS and ESRF for providing beamtime and facilities.

\section{References}

Baert, J., P. Van Puyvelde, and F. Langouche, "Flow-induced crystallization of PB-1: From the low shear rate region up to processing rates," Macromolecules 39(26), 9215-9222 (2006).

Becu, L., S. Manneville, and A. Colin, "Spatiotemporal dynamics of wormlike micelles under shear," Phys. Rev. Lett. 93(1), 018301(2004).

Bent, J., L. R. Hutchings, R. W. Richards, T. Gough, R. Spares, P. D. Coates, I. Grillo, O. G. Harlen, D. J. Read, R. S. Graham, A. E. Likhtman, D. J. Groves, T. M. Nicholson, and T. C. B. McLeish, "Neutron-mapping polymer flow: Scattering, flow visualization, and molecular theory," Science 301(5640), 1691-1695 (2003).

Berret, J. F., G. Porte, and J. P. Decruppe, "Inhomogeneous shear rows of wormlike micelles: A master dynamic phase diagram,” Phys. Rev. E 55(2), 1668-1676 (1997).

Caputo, F. E., W. R. Burghardt, K. Krishnan, F. S. Bates, and T. P. Lodge, "Time-resolved small-angle x-ray scattering measurements of a polymer bicontinuous microemulsion structure factor under shear," Phys. Rev. E 66(4), 041401(2002).

Carreras, E. S., J. M. Piau, N. El Kissi, F. Pignon, and P. Panine, "New capillary rheometer allowing for small-angle x-ray scattering experiments inside the die. Application to the extrusion of block copolymers, their macroscopic defects, and their structure," J. Rheol. 50(6), 803-829 (2006).

Chen, Z. R., J. A. Kornfield, S. D. Smith, J. T. Grothaus, and M. M. Satkowski, "Pathways to macroscale order in nanostructured block copolymers," Science 277(5330), 1248-1253 (1997).

Dean, D. M., L. Rebenfeld, R. A. Register, and B. S. Hsiao, "Matrix molecular orientation in fiber-reinforced polypropylene composites," J. Mater. Sci. 33(19), 4797-4812 (1998).

Eder, G., and H. Janeschitz-Kriegl, “ Crystallization," Processing of Polymers, edited by Meijer, H. E. H. (Wiley-VCH, New York, 1997), Vol. 18 pp. 269-342.

Eder, G., H. Janeschitzkriegl, and S. Liedauer "Crystallization processes in quiescent and moving polymer melts under heat-transfer conditions," Prog. Polym. Sci. 15(4), 629-714 (1990).

Fernandez-Ballester, L., "Formation of oriented precursors in flow-induced polymer crystallization: Experimental methods and model materials," Ph.D. thesis, California Institute of Technology, 2007.

Fernandez-Ballester, L., T. Gough, F. Meneau, W. Bras, F. Ania, J. C. Francisco, and J. A. Kornfield, "Simultaneous birefringence, small- and wide-angle X-ray scattering to detect precursors and characterize morphol- 
ogy development during flow-induced crystallization of polymers,” J. Synchrotron Radiat. 15, 185-190 (2008).

Fuller, G. G., Optical Rheometry of Complex Fluids (Oxford University Press, New York, 1995).

Fuller, G. G., "Optical rheometry of complex fluid interfaces," Curr. Opin. Colloid Interface Sci. 2(2), 153-157 (1997).

Hassell, D. G., and M. R. Mackley, "Localised flow-induced crystallisation of a polyethylene melt," Rheol. Acta 47(4), 435-446 (2008).

Hongladarom, K., W. R. Burghardt, S. G. Baek, S. Cementwala, and J. J. Magda, "Molecular alignment of polymer liquid-crystals in shear flows. 1. Spectrographic birefringence technique, steady-state orientation, and normal stress behavior in poly(benzyl glutamate) solutions," Macromolecules 26(4), $772-784$ (1993).

Janeschitz-Kriegl, H., and G. Eder, "Basic concepts of structure formation during processing of thermoplastic materials,” J. Macromol. Sci., Chem. A27(13-14), 1733-1756 (1990).

Janimak, J. J., S. Z. D. Cheng, P. A. Giusti, and E. T. Hsieh, "Isotacticity effect on crystallization and melting in poly(propylene) fractions. 2. Linear crystal-growth rate and morphology study," Macromolecules 24(9), 2253-2260 (1991).

Keller, A., and H. W. H. Kolnaar, "Flow-induced orientation and structure formation," Processing of Polymers, edited by Meijer, H. E. H. (Wiley-VCH, New York, 1997), Vol. 18, pp. 189-268.

Kimata, S., T. Sakurai, Y. Nozue, T. Kasahara, N. Yamaguchi, T. Karino, M. Shibayama, and J. A. Kornfield"Molecular basis of the shish-kebab morphology in polymer crystallization," Science 316(5827), 10141017 (2007).

Kornfield, J. A., G. Kumaraswamy, and A. M. Issaian, "Recent advances in understanding flow effects on polymer crystallization,” Ind. Eng. Chem. Res. 41(25), 6383-6392 (2002).

Krishnan, K., F. S. Bates, and T. P. Lodge, "Shear alignment of a swollen lamellar phase in a ternary polymer blend," J. Rheol. 49(6), 1395-1408 (2005).

Kumaraswamy, G., "Crystallization of polymers from stressed melts," J. Macromol. Sci., Part C Polym. Rev. 45(45), 375-397 (2005).

Kumaraswamy, G., A. M. Issaian, and J. A. Kornfield, "Shear-enhanced crystallization in isotactic polypropylene. 1. Correspondence between in situ rheo-optics and ex situ structure determination," Macromolecules 32(22), 7537-7547 (1999a).

Kumaraswamy, G., R. K. Verma, A. M. Issaian, P. Wang, J. A. Kornfield, F. Yeh, B. S. Hsiao, and R. H. Olley, "Shear-enhanced crystallization in isotactic polypropylene Part 2. Analysis of the formation of the oriented 'skin'," Polymer 41(25), 8931-8940 (2000).

Kumaraswamy, G., R. K. Verma, and J. A. Kornfield, "Novel flow apparatus for investigating shear-enhanced crystallization and structure development in semicrystalline polymers," Rev. Sci. Instrum. 70(4), 20972104 (1999b).

Kumaraswamy, G., R. K. Verma, J. A. Kornfield, F. J. Yeh, and B. S. Hsiao, "Shear-enhanced crystallization in isotactic polypropylene. In-situ synchrotron SAXS and WAXD," Macromolecules 37(24), 9005-9017 (2004).

Lagasse, R. R., and B. Maxwell, "Experimental-study of kinetics of polymer crystallization during shear-flow," Polym. Eng. Sci. 16(3), 189-199 (1976).

Langouche, F., "Orientation development during shear flow-induced crystallization of i-PP," Macromolecules 39(7), 2568-2573 (2006).

Lee, J. Y., G. G. Fuller, N. E. Hudson, and X. F. Yuan, "Investigation of shear-banding structure in wormlike micellar solution by point-wise flow-induced birefringence measurements," J. Rheol. 49(2), 537-550 (2005).

Liedauer, S., G. Eder, and H. Janeschitzkriegl, "On the limitations of shear-induced crystallization in polypropylene melts,” Int. Polym. Process. 10(3), 243-250 (1995).

Liedauer, S., G. Eder, H. Janeschitzkriegl, P. Jerschow, W. Geymayer, and E. Ingolic, "On the kinetics of shear-induced crystallization in polypropylene," Int. Polym. Process. 8(3), 236-244 (1993).

Nogales, A., B. S. Hsiao, R. H. Somani, S. Srinivas, A. H. Tsou, F. J. Balta-Calleja, and T. A. Ezquerra, "Shear-induced crystallization of isotactic polypropylene with different molecular weight distributions: In situ small- and wide-angle x-ray scattering studies," Polymer 42(12), 5247-5256 (2001). 
Norman, A. I., W. H. Zhang, K. L. Beers, and E. J. Amis, "Microfluidic light scattering as a tool to study the structure of aqueous polymer solutions," J. Colloid Interface Sci. 299(2), 580-588 (2006).

Pennings, A. J., and A. M. Kiel, "Fractionation of polymers by crystallization from solution. 3. On morphology of fibrillar polyethylene crystals grown in solution,” Kolloid Z. Z. Fur Polym. 205(2), 160-162 (1965).

Rendon, S., W. R. Burghardt, M. L. Auld, and J. A. Kornfield, "Shear-induced alignment of smectic side group liquid crystalline polymers," Macromolecules 40(18), 6624-6630 (2007).

Roe, R. J., Methods of X-ray and Neutron Scattering in Polymer Science (Oxford University Press, New York, 2000).

Seki, M., D. W. Thurman, J. P. Oberhauser, and J. A. Kornfield, "Shear-mediated crystallization of isotactic polypropylene: The role of long chain-long chain overlap," Macromolecules 35(7), 2583-2594 (2002).

Somani, R. H., B. S. Hsiao, A. Nogales, H. Fruitwala, S. Srinivas, and A. H. Tsou, "Structure development during shear flow induced crystallization of i-PP: In situ wide-angle X-ray diffraction study," Macromolecules 34(17), 5902-5909 (2001).

Somani, R. H., I. Sics, and B. S. Hsiao, "Thermal stability of shear-induced precursor structures in isotactic polypropylene by rheo-X-ray techniques with Couette flow geometry,” J. Polym. Sci., Part B: Polym. Phys. 44(24), 3553-3570 (2006).

Somani, R. H., L. Yang, L. Zhu, and B. S. Hsiao, "Flow-induced shish-kebab precursor structures in entangled polymer melts," Polymer 46(20), 8587-8623 (2005).

Thurman, D. W., Ph.D. thesis "Molecular aspects of flow-induced crystallization of polypropylene," California Institute of Technology, 2005.

Wales, J. L. S., "Apparatus for the measurement of flow birefringence of polymer melts at high shear," Rheol. Acta 8(1), 38-44 (1969). 